\title{
Counteracting tinnitus by acoustic coordinated reset neuromodulation
}

\author{
Peter A. Tass ${ }^{\mathrm{a}, \mathrm{b}, *}$, Ilya Adamchic ${ }^{\mathrm{a}}$, Hans-Joachim Freund ${ }^{\mathrm{a}}$, Tatjana von Stackelberg ${ }^{\mathrm{c}}$ \\ and Christian Hauptmann ${ }^{\mathrm{a}}$ \\ ${ }^{a}$ Research Center Jülich, Institute for Neuroscience and Medicine - Neuromodulation INM-7, Jülich, Germany \\ ${ }^{\mathrm{b}}$ Department of Stereotaxic and Functional Neurosurgery, University Hospital, Cologne, Germany \\ ${ }^{\mathrm{c}}$ Ear, Nose and Throat (ENT) Center, Meerbusch, Germany
}

\begin{abstract}
Purpose: Subjective tinnitus is associated with pathologic enhanced neuronal synchronization. We used a model based desynchronization technique, acoustic coordinated reset (CR) neuromodulation, to specifically counteract tinnitus-related neuronal synchrony thereby inducing an unlearning of pathological synaptic connectivity and neuronal synchrony.

Methods: In a prospective, randomized, single blind, placebo-controlled trial in 63 patients with chronic tonal tinnitus and up to $50 \mathrm{~dB}$ hearing loss we studied safety and efficacy of different doses of acoustic CR neuromodulation. We measured visual analogue scale and tinnitus questionnaire (TQ) scores and spontaneous EEG.

Results: CR treatment was safe, well-tolerated and caused a significant decrease of tinnitus loudness and symptoms. Placebo treatment did not lead to any significant changes. Effects gained in 12 weeks of treatment persisted through a preplanned 4-week therapy pause and showed sustained long-term effects after 10 months of therapy: Response, i.e. a reduction of at least 6 TQ points, was obtained in $75 \%$ of patients with a mean TQ reduction of $50 \%$ among responders. CR therapy significantly lowered tinnitus frequency and reversed the tinnitus related EEG alterations.

Conclusion: The CR-induced reduction of tinnitus and underlying neuronal characteristics indicates a new non-invasive therapy which might also be applicable to other conditions with neuronal hypersynchrony.
\end{abstract}

Keywords: Tinnitus treatment, desynchronization, plasticity, non-invasive neuromodulation

\section{Introduction}

Subjective tinnitus is an acoustic phantom phenomenon, a perception of sound in the absence of physical sound sources (Eggermont, 2003; Lockwood et al., 2002; Moller, 2003; Weisz et al., 2005a). This type of tinnitus is typically initiated by damage to the peripheral hearing system (Irvine et al., 2001; Lockwood et al., 2002; Norena et al., 2002;

${ }^{*}$ Corresponding author: Prof. Dr. Peter A. Tass, Research Center Jülich, Institute for Neuroscience and Medicine - Neuromodulation INM-7, 52425 Jülich, Germany. Tel.: +49 246161 2087; Fax: +49 246161 1880; E-mail: p.tass@fz-juelich.de.
Weisz et al., 2006) leading to a sequence of structural and functional changes in the central hearing system (Eggermont, 2003; Lockwood et al., 2002; Moller, 2003; Eggermont and Roberts, 2004; Saunders, 2007). Both human and animal data show that deafferentation alters receptive fields (Rajan and Irvine, 1998; Rauschecker, 1999; Irvine et al., 2001; Dietrich et al., 2001) and spontaneous activity (Eggermont, 2003; Norena and Eggermont, 2003; Elbert et al., 1997) of neurons in the auditory cortex. There are two major phenomena associated with tinnitus: cortical map reorganization (Robertson and Irvine, 1989; Mühlnickel et al., 1998; Lockwood et al., 2002; Moller, 2003) and the emergence of pathological neural synchrony 
(Ochi and Eggermont, 1997; Norena and Eggermont, 2003; Seki and Eggermont, 2003; Weisz et al., 2005a; Weisz et al., 2007).

Cortical map reorganization was demonstrated in animals with restricted cochlear lesions (Robertson and Irvine, 1989; Rajan and Irvine, 1998; Rauschecker, 1999; Irvine et al., 2001) and in tinnitus patients (Mühlnickel et al., 1998; Dietrich et al., 2001; Weisz et al., 2005b). In a magnetoencephalography (MEG) study Mühlnickel et al. (1998) found that the cortical representation of the tinnitus frequency was clearly shifted into an area adjacent to the expected tonotopic location. However, patients with audiometrically impaired hearing were excluded from that study, so that subjects were not representative of typical tinnitus sufferers. In contrast, recent results obtained with MEG recordings in tinnitus patients suggest that mechanisms of map reorganization cannot explain the emergence of tinnitus in a satisfactory manner (Weisz et al., 2005a).

Pathologically increased $\delta$ activity emerges in cortical regions deprived of afferent input (Steriade, 2006). Correspondingly, MEG studies in patients with chronic subjective tinnitus revealed an increase of power in particular frequency bands (Llinás et al., 1999; Weisz et al., 2005a; Weisz et al., 2007). An increase of band power in MEG, electroencephalogram (EEG) and local field potential (LFP) signals is typically interpreted as an increase in neuronal synchronization in terms of coincident firing within a neuronal population (see Klaas and Daly, 1979; Nunez, 1981; Hämäläinen et al., 1993; Niedermeyer and Lopes da Silva, 1999 and Discussion). In an MEG study Weisz et al. (2005a) showed that in tinnitus patients $\alpha$ band power was significantly reduced, whereas $\delta$ band power was significantly increased, particularly in temporal regions. Furthermore, tinnitus related distress was correlated with this abnormal pattern of spontaneous activity in particular in right temporal and left frontal brain areas. In a further MEG study Weisz et al. (2007) analyzed $\gamma$ band activity during brief epochs of pronounced enhancement of $\delta$ wave activity. In both groups, controls and tinnitus patients, $\gamma$ band activity was significantly increased after onset of $\delta$ waves. However, $\gamma$ was more pronounced in tinnitus subjects compared to controls. $\gamma$ activity at $55 \mathrm{~Hz}$ was related to the laterality of the tinnitus perception. Pathological neural synchronization has consistently been confirmed in further studies as the electrophysiological correlate of the tinnitus percept. Tinnitus could be reduced by an EEG neurofeedback mediated suppression of $\delta$ band activity and enhancement of $\alpha$ band (Dohrmann et al., 2007a). In an MEG study a significant reduction of $\delta$ band activity in temporal areas was found during residual inhibition (Kahlbrock and Weisz, 2008). Epidural recordings from the secondary auditory cortex in a tinnitus patient revealed abnormal $\theta$ band activity highly correlated with tinnitus loudness (De Ridder et al., 2011). Also in patients with acute tinnitus after noise trauma abnormal neuronal synchronization, namely increased $\gamma$ band activity in the right auditory cortex, was found in an MEG study (Ortmann et al., 2011). Not only auditory cortical areas are involved in the generation of tinnitus. Rather there is growing evidence that non-auditory areas, e.g., amygdala, cingulate cortex and parahippocampus, play a crucial rule, in particular, in patients with relevant tinnitus distress (Rauschecker et al., 2010; Vanneste et al., 2010). In fact, the functional interaction between non-auditory and auditory areas displaying abnormal synchrony appears to be a central feature underlying tinnitus distress (Schlee et al., 2009a).

As yet, typically used tinnitus treatment strategies, such as tinnitus retraining therapy (Jastreboff and Hazell, 1993), are basically approaches to facilitate habituation. In fact, there is a great demand for more causally oriented treatments specifically targeting the neuronal correlates of the tinnitus percept. Different approaches have been tested so far (Moller et al. 2011). Depending on the parameters chosen, transcranial magnetic stimulation (TMS) may lead to a decrease (Chen et al., 1997) or increase (PascualLeone et al., 1994) of cortical excitability along with long-term potentiation or long-term depression (Wang et al., 1996). TMS has been applied to tinnitus patients (Plewnia et al., 2003; De Ridder et al., 2005). Although in a subgroup of patients repetitive TMS resulted in tinnitus relief, the effects were only moderate, whereas interindividual variability is high, so that further improvement is required until TMS can be recommended for clinical use (Kleinjung et al., 2011). Another intervention with quite complex and still not sufficiently understood mechanism of action is auditory cortex stimulation (De Ridder et al., 2005; De Ridder et al., 2006). One mechanism of action may be that cortical stimulation directly provides deafferented cortex with missing input (De Ridder et al., 2007). One in three patients responds best to tonic auditory cortex stimulation, and one in three to burst stimulation (De Ridder et al., 2011; De Ridder and Vanneste, 2011). 
Unlike this invasive approach, neurofeedback for the treatment of tinnitus aims at a suppression of pathological patterns of synchrony, e.g., a suppression of $\delta$ along with an enhancement of $\alpha$ power (Dohrmann et al., 2007a). To be used for clinical purposes, neurofeedback approaches still require improvement (Hartmann et al., 2011).

Apart from direct cortical stimulation (De Ridder et al., 2005; De Ridder et al., 2006), further approaches have been chosen to specifically counteract cortical map reorganization, e.g. by means of acoustic stimulation with tailor-made notched music (Okamoto et al., 2010) or by means of vagus nerve stimulation (VNS) combined with multiple tone pairing, an invasive therapy tested so far in animals only (Engineer et al., 2011).

However, since several studies showed that pathological neuronal synchronization is highly correlated with tinnitus loudness (Weisz et al., 2005a; Dohrmann et al., 2007a; Kahlbrock and Weisz, 2008; van der Loo et al., 2009; De Ridder et al., 2011), whereas the relationship between cortical map reorganization and subjective measures of tinnitus is not consistent (Mühlnickel et al., 1998; Weisz et al., 2005b; Yang et al., 2011), we here use a different approach. To reduce tinnitus symptoms, we specifically counteract the electrophysiological correlate of tinnitus, pathological neural synchrony, by a desynchronization technique. Our approach employs the intimate relationship between neuronal dynamics and connectivity (Yuste and Bonhoeffer, 2004). As shown computationally, coordinated reset (CR) stimulation causes a desynchronization (Tass, 2003) shifting a network comprising spike timing-dependent plasticity (Gerstner et al., 1996; Markram et al., 1997) from a synchronized state with strong synaptic connectivity to a desynchronized state with weak connectivity (Tass and Majtanik, 2006; Hauptmann and Tass, 2007; Tass and Hauptmann, 2009): The network undergoes an anti-kindling, i.e. it unlearns pathological connectivity and pathological synchrony. CR neuromodulation was initially developed for electrical deep brain stimulation (DBS) in Parkinson's disease (Tass, 2003), where brief electrical stimuli are sequentially delivered through different contacts of an implanted lead. Both, acute desynchronizing effects (Neiman et al., 2007) and long-lasting desynchronizing after-effects (Tass et al., 2009) of electrical CR have been verified in animal experiments.

We here transform in this first in man trial the CR concept into a non-invasive, acoustic treatment for subjective tonal tinnitus. To desynchronize a synchronized focus in the tonotopically-organized auditory cortex located in an area corresponding to the dominant tinnitus frequency, we acoustically delivered four $\mathrm{CR}$ tones with different frequencies centered around the patient's individual tinnitus frequency (Fig. 1). The intent of each CR tone is to induce a phase reset or at least a soft phase reset (Tass, 2002), i.e. a phase reset achieved by an iterated delivery of that CR tone, of the pathological slow-wave oscillation in the $\delta$ frequency band in a sub-population of neurons tonotopically related to the tone's particular frequency. A sequence of $\mathrm{CR}$ tones induces a robust desynchronization as shown numerically in the context of both DBS (Tass, 2003; Tass and Hauptmann, 2009) and acoustic CR neuromodulation (Tass and Popovych, 2012). Based on our previous computational (Tass, 2003; Tass and Majtanik, 2006; Hauptmann and Tass, 2007; Tass and Hauptmann, 2009; Hauptmann and Tass, 2009; Hauptmann and Tass, 2010; Tass and Popovych, 2012) and animal studies (Neiman et al., 2007; Tass et al., 2009) we expected to find a long-lasting tinnitus relief along with a long-lasting desynchronization of tinnitusrelated pathological neural synchrony. Acoustic CR neuromodulation had not yet been applied to animals or humans. By the same token, the mechanism of acoustic CR neuromodulation (i.e. desynchronization achieved by a sequential phase reset of neuronal subpopulations) had not yet been studied in animals or humans.

\section{Materials and methods}

\subsection{Study subjects}

The RESET study was a first in man dose finding trial with a prospective, single blind, multi-center, randomized, placebo-controlled, parallel group study design ("RESET study", NCT00927121, EC Freiburg, Germany). 99 patients with tinnitus were screened in 8 centers; 36 patients did not fulfill the inclusion criteria, mainly due to presence of atonal tinnitus or tinnitus duration less than 6 months. 26 and 37 patients were enrolled at two treatment centers, respectively. These 63 patients ( $\geq 18$ years) with chronic ( $\geq 6$ months), tonal (reliable and repeated location of the tinnitus tone by a sinusoidal matching tone), subjective tinnitus, not engaged in other tinnitus therapy and able to hear the stimulation tones and up to moderate hearing impairment (up to $50 \mathrm{~dB}$ within the frequency 


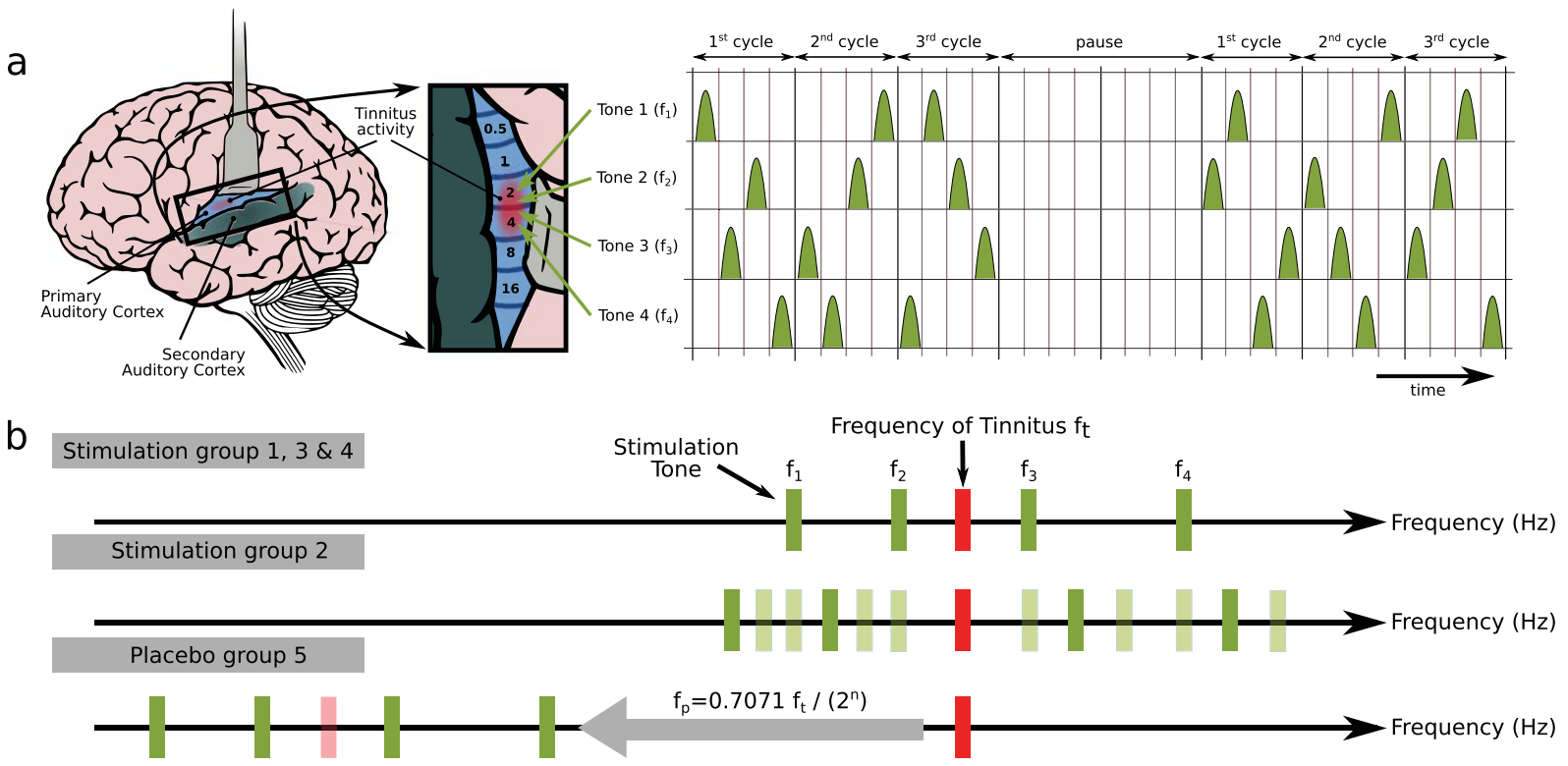

Fig. 1. Acoustic coordinated reset (CR) neuromodulation: (a) The concept of CR neuromodulation comprises a spatial and temporal coordination of the applied stimuli to induce desynchronization leading to anti-kindling (Tass, 2003): utilizing the tonotopic organization of the primary auditory cortex (left, brain adapted from Chittka and Brockmann PLoS Biology, 2005 with kind permission of the authors) short sinusoidal tones of different frequencies ( $f_{1}$ to $f_{4}$ ) induce a soft reset in different target areas grouped around the tinnitus focus (Tass, 2002). Three CR cycles, each comprising a randomized sequence of four tones (right), are followed by two silent cycles. That pattern is repeated periodically. The random variation of the tone sequences (Tass and Majtanik, 2006) and the 3:2 ON-OFF pattern (Tass, 2003, Lysyansky et al., 2011) optimize the desynchronizing CR effect. (b) Four stimulation groups and one placebo group were investigated. For G1, G3 and G4 four tones (top, $\mathrm{f}_{1}$ to $\mathrm{f}_{4}$ ) are grouped around the tinnitus frequency $\left(\mathrm{f}_{\mathrm{t}}\right)$. G3 differs only in repetition rate $\mathrm{F}$ being adapted to the individual EEG $\delta$-band peak. For $\mathrm{G} 2$ each CR cycle is formed by a varying composition of four tones (dark green: active) chosen out of twelve tones (middle, $f_{1}$ to $f_{12}$ ) surrounding $f_{t}$. Placebo stimulation (bottom, G5) is formed similar to G1 using a down-shifted stimulation-frequency $f_{p}\left(f_{p}=0.7071 \cdot f_{t} /\left(2^{n}\right), f_{p}\right.$ within $[300 ~ H z$, $600 \mathrm{~Hz}]$ ) outside the synchronized tinnitus focus.

band of the stimulation tones measured by an audiogram from 0.125 to $12 \mathrm{kHz}$ ) were randomly allocated to receive CR neuromodulation (group G1 to G4) or placebo stimulation (G5): G1 $(n=22), \mathrm{G} 2(n=12), \mathrm{G} 3$ $(n=12), \mathrm{G} 4(n=12)$, G5 $(n=5)$, see Table 1. Central randomization was provided by a clinical research organization (CRO). The randomization scheme was computer generated utilizing a block randomization. The assignment of treatments occurred in accordance with the numerical sequence of patient enrolment using sealed envelopes. A higher number of patients was allocated to G1 to corroborate the database for its suspected most efficacious stimulation algorithm.

Exclusion criteria were: Morbus Meniere, auditory hallucinations, symptomatic hearing disorders, tinnitus due to temporomandibular joint disorders, brainstem diseases, psychiatric disorders and objective tinnitus. The ethical committee approved the trial design and all changes. The safety of the study was monitored by an independent Data and Safety Monitoring Board (DSMB). The study was performed in accordance with the Declaration of Helsinki and Good Clinical Practice guidelines. All participating patients gave their written informed consent.

\subsection{Tinnitus characteristic analysis}

The tinnitus frequency $f_{t}$ (from $100-10.000 \mathrm{~Hz}$ ) was assessed with a pure tone matching, where intensity and frequency of the matching tone were controlled by the patient.

\subsection{Study design}

Patients were stimulated for 12 weeks using a portable acoustic device and comfortable earphones (loudness controlled by patient) followed by an additional off-stimulation 4-week period to assess lasting 
Table 1

Baseline data

\begin{tabular}{|c|c|c|c|c|c|}
\hline & $\mathrm{Gl}(n=22)$ & $\mathrm{G} 2(n=12)$ & $\mathrm{G} 3(n=12)$ & $\mathrm{G} 4(n=12)$ & G5 $(n=5)$ \\
\hline Age $[$ mean $(\mathrm{SD})]$ & $45.7(10.8)$ & $47.7(5.6)$ & $50.0(14.7)$ & $50.3(11.8)$ & $57.6(6.3)$ \\
\hline Sex (male \%) & 72.7 & 83.3 & 50.0 & 75.0 & 60.0 \\
\hline $\mathrm{BMI}\left(\mathrm{kg} / \mathrm{m}^{2}\right)$ [mean (SD)] & $26.1(5.2)$ & $27.7(4.2)$ & $26.8(4.3)$ & $25.7(3.6)$ & $25.3(4.0)$ \\
\hline Smoke (yes \%) & 27.3 & 25.0 & 16.7 & 0.0 & 40.0 \\
\hline Social drinking (yes \%) & 77.3 & 66.7 & 66.7 & 75.0 & 100.0 \\
\hline Tinnitus duration (in years) [mean (SD)] & $5.7(5.1)$ & $6.6(6.0)$ & $5.4(3.5)$ & $7.9(9.8)$ & $11.3(5.6)$ \\
\hline Tinnitus side (unilateral \%) & 45.5 & 33.3 & 50.0 & 16.7 & 40.0 \\
\hline VAS Loudness [mean (SD)] & $70.9(14.9)$ & $52.1(21.5)$ & $66.3(20.4)$ & $72.1(16.7)$ & $43.0(19.2)$ \\
\hline VAS Annoyance [mean (SD)] & $66.4(17.3)$ & $54.2(21.6)$ & $64.2(22.0)$ & $70.8(17.0)$ & $38.0(14.8)$ \\
\hline $\mathrm{TQ}[$ mean (SD)] & $43.1(16.7)$ & $38.6(15.9)$ & $36.5(16.4)$ & $50.8(15.9)$ & $29.2(7.7)$ \\
\hline Tinnitus frequeny $(\mathrm{kHz})$ [mean $(\mathrm{SD})]$ & $7.7(2.9)$ & $5.0(2.5)$ & $4.7(3.5)$ & $5.4(3.3)$ & $5.8(3.4)$ \\
\hline EEG $\delta$ power (a.u.) $[$ mean (SD)] & $0.23 \oint(0.11)$ & $0.27 £(0.13)$ & $0.26(0.09)$ & $0.22(0.10)$ & $0.24(0.1)$ \\
\hline EEG $\alpha$ power (a.u.) [mean (SD)] & $0.37 \AA(0.2)$ & $0.32 £(0.19)$ & $0.34(0.21)$ & $0.36(0.17)$ & $0.35(0.17)$ \\
\hline
\end{tabular}

§EG analysis: $n=21$ (11) were evaluable in G1 (G2).

effects of acoustic CR neuromodulation; an optional open-label long-term extension (LTE) period (24 weeks) was offered. Visits took place 1, 4, 8, 12 and 16 weeks after beginning of treatment and every 4 weeks during LTE.

Tinnitus loudness and annoyance were first measured off-stimulation (at least 2.5 hours after

\subsection{Euclidean distance of normalized clinical scores}

The Euclidean distance was calculated based on the normalized clinical scores $\left(\mathrm{VAS}_{\text {Loudness }}, \mathrm{VAS}_{\text {Annoyance }}\right.$ and TQ). The Euclidean distance d between two subgroups of $n$ patients was defined as

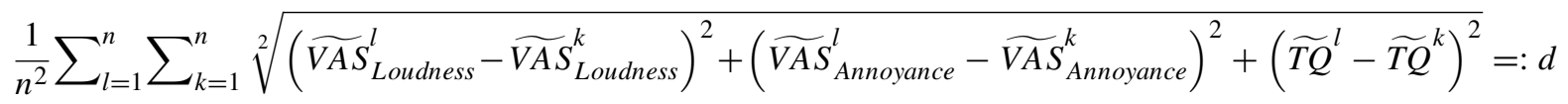

where $\widetilde{\operatorname{VAS}}_{\text {Loudness }}^{1}\left(\widetilde{\mathrm{VAS}}_{\text {Loudness }}^{\mathrm{k}}\right)$ denote the normalized value of the $\mathrm{VAS}_{\text {Loudness }}$ score for patient $1(\mathrm{k})$ from

cessation of CR neuromodulation) and consecutively on-stimulation (15 min after turning on CR neuromodulation) by a visual analog scale (VAS). VAS scales ranged from 0 (corresponding to absence of tinnitus) to 100 (corresponding to maximal imaginable loudness or annoyance). The tinnitus distress level was captured by the German version of the Tinnitus Questionnaire (TQ, measured off-stimulation) (Goebel and Hiller, 1994). The TQ is a patient reported measure of 52 items assessing emotional and cognitive distress, intrusiveness, auditory perceptual difficulties, sleep disturbances and associated somatic complaints. The TQ is a highly reliable (test-retest reliability 0.94 ) and valid instrument with scores ranging from 0 to 84 (Goebel and Hiller, 1998). Like the VAS loudness and VAS annoyance, higher scores on the TQ reflect greater severity. Mean values and standard deviations are given. Auditory thresholds were measured by an audiogram (performed at $0.125,0.25,0.5$, $0.75,1,1.5,2,3,4,6,8,12 \mathrm{kHz})$ in a sound-proof chamber. the first (second) subgroup, respectively (similarly for VAS $_{\text {Annoyance }}$ and TQ). Normalization, i.e. division by the maximal value, was performed in order to achieve a uniform weighting of all three clinical scores.

\subsection{Stimulation protocol}

G1 to G3 all received stimulation for 4-6 hours every day (Fig. 1b) applied either continuously or split into several sessions not shorter than 1 hour each to utilize cumulative effects (Jacobson and Truax, 1991); G4 and G5 all received stimulation for 1 hour max. every day. Stimulation signals were generated based on a specific formula reflecting the logarithmic tonotopic organization of the auditory cortex and on the matched tinnitus (frequency $f_{t}$ ) with an equal number of tones placed below and above tinnitus frequency (except for placebo, Fig. 1b). Stimulation tones were equally loud and just super-threshold. 4 tones per cycle were played in random order with 3 stimulation cycles followed by 
2 silent cycles (Fig. 1a). The silent cycles were introduced in order to optimize the duration of the transient desynchronization and, hence, the anti-kindling effect (see Tass, 2003; Lysyansky et al., 2011). The 4 tones are based on a patient specific list of frequencies, see Fig. 1: $\mathrm{G} 1, \mathrm{G} 3, \mathrm{G} 4$ ( $\mathrm{f}_{1}$ to $\left.\mathrm{f}_{4}\right)$; $\mathrm{G} 2$ (prior to each cycle 4 frequencies are chosen from $\mathrm{f}_{1}$ to $\left.\mathrm{f}_{12}\right)$; $\mathrm{G} 5$ based on a modified tinnitus frequency $\left(f_{p}=0.7071 f_{t} /\left(2^{n}\right), f_{p}\right.$ within 300 to $600 \mathrm{~Hz}$ ). The stimulation tones are equidistantly placed on a logarithmic scale within the interval $\left[0.5 \cdot f_{t}, 2 \cdot f_{t}\right]$ for G1 to G4 and within $\left[0.5 \cdot f_{p}, 2 \cdot f_{p}\right]$ for G5. Cycle repetition rate was $1.5 \mathrm{~Hz}$, i.e. in the lower $\delta$ frequency range, for G1, G2, G4, G5, because the primary target for desynchronization was the pathological $\delta$ band activity. We have chosen the repetition rate within the lower $\delta$ frequency range because according to theoretical studies it is more favorable to use a stimulation rate that is slightly to low instead of being slightly too high (see Tass, 2003; Lysyansky et al., 2011). Also, psychophysically greater repetition rates were perceived as less relaxing and, hence, less convenient. In contrast, in $\mathrm{G} 3$ the repetition rate was set equal to the peak frequency in the patient's $\delta$ frequency range according to the EEG data (Tass, 2003; Tass et al., 2009).

A readjustment of stimulation parameters could be done at each visit if matched tinnitus frequency had changed.

\subsection{EEG data}

Every patient underwent two EEG recording sessions: on the first treatment day before treatment began and at the 12 weeks visit, at least two hours after the end of the last stimulation session. EEG recordings were obtained in a Faraday cage with a 128 EEG surface electrodes system (128 channel HydroCel Geodesic Sensor Net) in a quiet and dimly lighted room with each participant sitting upright on a comfortable chair. All electrodes were referenced to Cz. Recordings were performed twice in awake patients with eyes closed and eyes open for $2 \mathrm{~min}$ each. We have selected eyes closed data for further analysis since they were less affected by artifacts. Impedances were kept below 100 $\mathrm{k} \Omega$ as required by the EEG recording system. EEG signals were digitized at $1 \mathrm{kHz}$ and digitally filtered with a $0.8-130 \mathrm{~Hz}$ digital filter. Each EEG recording was corrected for blink and eye movements in BESA using a surrogate model approach from BESA (Scherg et al., 2002) (Brain Electrical Source Analysis, MEGIS Software, version 5.2). Recordings were further analyzed in MATLAB (The Mathworks, Natick, MA) using EEGLAB (http://sccn.ucsd.edu/eeglab) where muscle artifacts and artifacts from heart activity (electrocardiogram) were removed. The total eyes closed recording after artifact correction lasted on average $3 \mathrm{~min} 36 \mathrm{sec} \pm 24 \mathrm{sec}$. Two types of inverse calculation of the surface EEG signals were performed: (i) Surface EEG was transformed into brain source activity of the primary auditory cortex (ACI, Brodmann area 41) using the source montage approach in BESA for G1 ( $n=21$ evaluable) and G5 $(n=5)$. According to previous studies (Mühlnickel et al., 1998; Lockwood et al., 1998; Lockwood et al., 1999; Melcher et al., 2000; Kovacs et al., 2006; Smits et al., 2007; Weisz et al., 2007; van der Loo et al., 2009) in patients with unilateral tinnitus we used the contralateral ACI, in patients with bilateral tinnitus we used both ACI. (ii) In patients with bilateral tinnitus surface EEG was transformed into current source density with SLORETA (Pascual-Marqui, 2002), where the three-dimensional solution space was restricted to the cortical gray matter. Unilateral and bilateral tinnitus patients can have different EEG abnormalities (Vanneste et al., 2011). Accordingly, to avoid an influence of such differences, we selected only patients with bilateral tinnitus $(n=28)$ from $\mathrm{G} 1$ to $\mathrm{G} 4$. To increase the signal-tonoise ratio from this population of 28 patients with bilateral tinnitus 12 patients were selected for the sLORETA analysis following the TQ based reliablechange-index (RCI) method (Jacobson and Truax, 1991). This subgroup of $n=12$ patients had the following demographic and clinical characteristics (mean (SD)): age 46.5 (7.9), tinnitus duration 7.6 (7.4), TQ at baseline 52.3 (17.5), $\mathrm{VAS}_{\text {Loudness }}$ at baseline 72.1

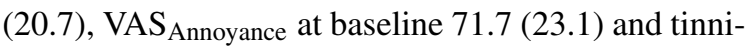
tus frequency $5.9 \mathrm{kHz}(3.5)$. The sLORETA subgroup showed the following improvements at 12 weeks: TQ -19.5 (9.1), VAS $_{\text {Loudness }}$ on/off stimulation -37.9 (35.4)/-26.7 (26.2), VAS $_{\text {Annoyance on/off stimulation }}$ $-41.3(35.2) /-21.3$ (26.6). Images were transformed to the stereotactic Talairach space and overlaid with a standard, structural MRI scan (MNI152 template). Statistical significance of sLORETA changes was nonparametrically assessed on a voxel-by-voxel basis with a randomization test (Nichols and Holmes, 2002) which corrects for multiple comparisons. Finally, power spectra were calculated for both types of inverse solutions and averaged across patients. Power spec- 
tra, calculated using fast Fourier transform, were divided into normalized EEG frequency bands: $\delta$ $(1-4 \mathrm{~Hz}), \theta(4-8 \mathrm{~Hz}), \alpha(8-12 \mathrm{~Hz}), \beta(12-30 \mathrm{~Hz}), \gamma_{\text {low }}$ $(30-48 \mathrm{~Hz})$ and $\gamma_{\text {high }}(52-90 \mathrm{~Hz})$. Power spectra were averaged across patients, and frequency bands group differences between the untreated and treated conditions were tested with the Wilcoxon signed-rank test.

\subsection{Statistical methods}

The statistical analysis was based on an intentionto-treat analysis including all randomized subjects. The sample size estimation was based on pre-clinical observations and empirical considerations. For missing values the LOCF method was used (first 16 weeks).

Descriptive statistics were used to compare baseline to end-of-treatment values (Wilcoxon matched pairs performed on non-log-transformed data and Sign test). Comparison to placebo was done applying the Mann-Whitney $U$ test. Due to the exploratory nature we tested two-sided without adjustment for multiple comparisons at the 5\% significance level. Correlation was calculated using the Pearson product-moment correlation.

For sLORETA we assessed statistical significance, corrected for multiple comparisons, by means of a nonparametric randomization test (Nichols and Holmes, 2002).

\section{Results}

Clinical examination after 12 weeks of therapy revealed a strong (29.6 to 37.3 points) and significant reduction of VAS loudness/annoyance in G1 and G3 in the on-stimulation condition $(p \leq 0.01$ compared to baseline, Fig. 2a, Table 2). The effects in G1 are significant also compared to placebo $(p<0.05$, Fig. 2b). In the off-stimulation condition significant effects persisted for G1/G3 VAS loudness/annoyance (18.0 to 28.8 points, $p<0.004$ Fig. 2 a, Table 2 ) in accordance with theoretically predicted lasting antikindling effects. In G2, the noisy CR group, only on-stimulation effects showed significant reductions ( $p<0.05$, Fig. 2a, Table 2) and the difference between on- and off-stimulation effect was strongest (Fig. 2c, $p<0.01$ ). In G4 (reduced stimulation time of $1 \mathrm{~h} /$ day) we observed a significant decrease for both VAS loud- ness/annoyance scores in the on-stimulation condition $(p<0.05)$ and markedly less in off-stimulation (Fig. 2a, Table 2). In contrast, the placebo group G5 showed neither on- nor off-stimulation significant changes in VAS loudness/annoyance scores after 12 weeks, (Fig. 2a, Table 2).

After a subsequent 4-week therapy pause G1 and G3 preserved significant reductions in tinnitus loudness and annoyance (Fig. 2a, Table 2). In general, we found a high correlation between the CR induced changes in VAS loudness ( $\left.\triangle \mathrm{VAS}_{\text {Loudness }}\right)$ and annoyance $\left(\Delta \mathrm{VAS}_{\text {Annoyance }}\right)$ i.e. $r=0.92$ (baseline to 12 weeks, $p<0.001$, Fig. 2f).

$T Q$ severity levels significantly improved after 12 and 16 weeks in $\mathrm{G} 1$ ( $p<0.01$ compared to baseline) and G3 ( $p<0.05$, Fig. 2d, Table 2). In G4, a small but significant decrease of the TQ severity levels was seen after 12 weeks ( $p<0.05$, Fig. 2d, Table 2). G5 (placebo) did not show any significant change.

$T Q$ scores (mean values) were significantly reduced compared to baseline in G1 to G4 with the strongest improvements in G1 and G3 (12/16 weeks, Table 2). In contrast, there were no significant changes in TQ score in the placebo group G5 (Table 2).

Tinnitus frequency (pure tone matching) was significantly reduced in G1 after 12 and 16 weeks $(p<0.01$, Table 2), while placebo did not show significant changes of tinnitus frequency.

In all patients with a hearing impairment it was of a sensorineural type. Auditory threshold averaged from the three frequencies of our audiogram procedure (see methods) closest to the tinnitus frequency was $29.8 \mathrm{~dB}$. No significant changes in auditory thresholds were detected pre/post treatment.

\subsubsection{Impact of tinnitus duration, tinnitus severity and age on treatment outcome}

Analyzing for tinnitus duration, tinnitus severity and age revealed no confounding factor for treatment success of 4-6h/day treated CR neuromodulation (CR groups $\mathrm{G} 1+\mathrm{G} 3$ after 12 weeks tinnitus duration in years versus relative TQ change $r=0.14, p=0.43$, tinnitus severity in TQ versus relative TQ change $r=-0.16, p=0.35$ and age versus relative TQ change $r=0.09, p=0.62$; after 40 weeks for all patients tinnitus duration in years versus relative TQ change $r=-0.05$, $p=0.74$, tinnitus severity in TQ versus relative TQ change $r=0.18, p=0.19$ and age versus relative TQ change $r=0.01, p=0.94)$. 

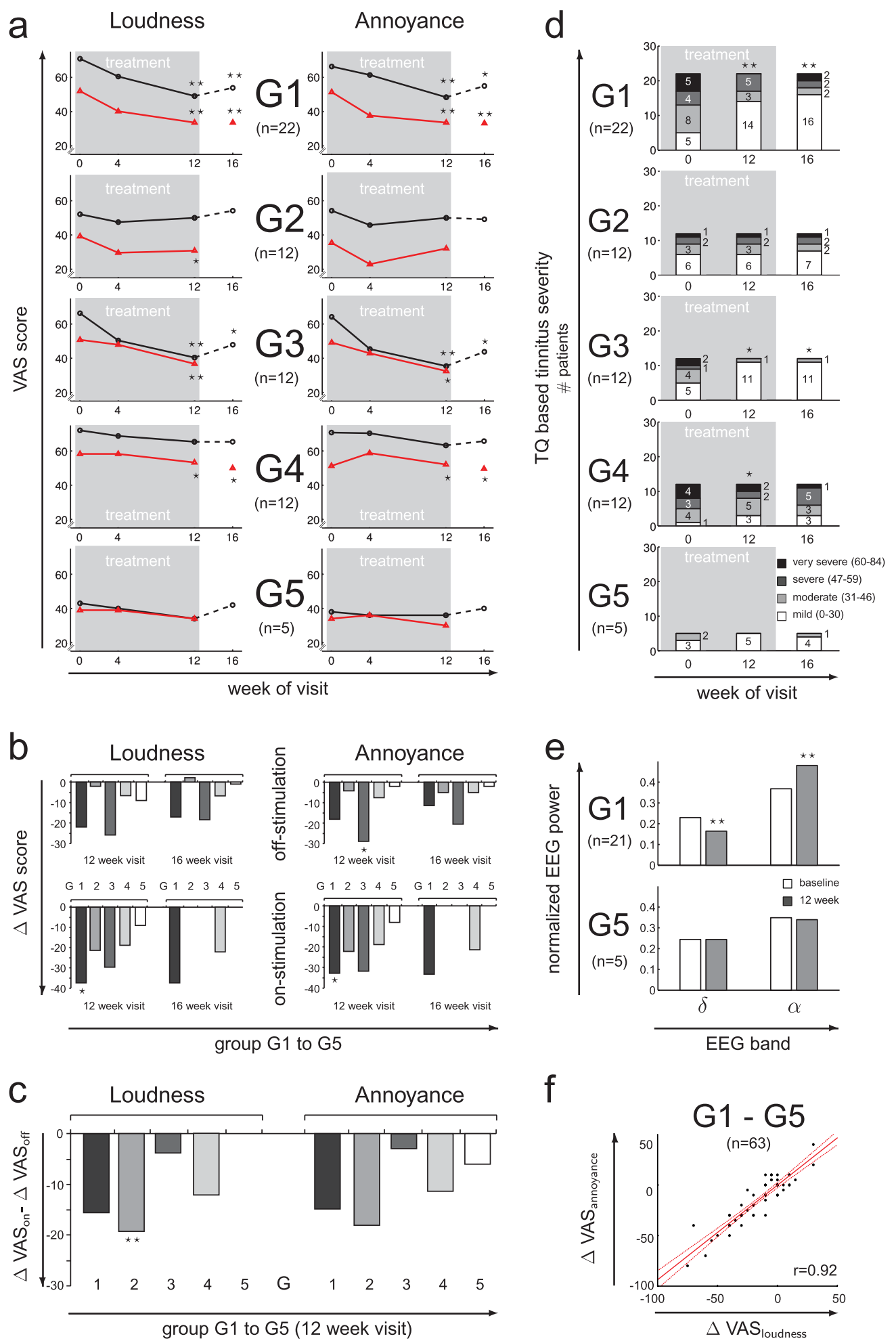

Fig. 2. (Continued) 


\subsubsection{Comparison between placebo group and equally sized, matched subgroups of $G 1$}

To better control for the baseline characteristics of the placebo group we matched G5 $(n=5)$ with an equally sized subgroup of G1 with comparable baseline characteristics (mean TQ exactly 29.2 pts in both groups at baseline and VAS scales for loudness and annoyance also not significantly different). We found a significant improvement compared to baseline (offstimulation) after the first 12 weeks of treatment for the G1 subgroup only (VAS loudness G1 sub -24 pts. mean, $p=0.07$, vs. G5 $-9 \mathrm{y}$ pts. mean, not significant; VAS annoyance G1 sub -23 pts. mean, $p=0.04$ vs. G5 -2 pts. mean, not significant; $\Delta$ TQ G1 sub -11.8 pts. mean, $p=0.04$, vs. G5 -8.4 pts. mean, not significant).

Furthermore we matched G5 $(n=5)$ with 100 equally sized subgroups of G1 with comparable baseline characteristics (mean TQ exactly 32.4 pts. at baseline ( $p=0.67$ as compared to G5) and VAS scales for loudness and annoyance also not significantly different). These G1 subgroups were selected from the 26334 possible combinations of 5 patients in the G1 subgroup derived from 22 patients in G1 based on the shortest Euclidean distance between each G1 sub group and G5. Euclidean distances were calculated based on the VAS loudness/annoyance and TQ for each G1 sub group separately (see Methods). We found a substantial improvement as compared to baseline (off-stimulation) after the first 12 weeks of treatment for the G1 subgroup (VAS loudness G1 sub -22 pts. mean, $p=0.08$, vs. G5
$-9 \mathrm{y}$ pts. mean, $p=0.50$; VAS annoyance G1 sub -19 pts. mean, $p=0.08$ vs. G5 -2 pts. mean, $p=1.00 ; \Delta \mathrm{TQ}$ $\mathrm{G} 1 \mathrm{sub}-9.5$ pts. mean, $p=0.06$, vs. G5 -8.4 pts. mean, $p=0.12)$.

\subsubsection{Pooled comparison between "effective" and "ineffective" stimulation groups}

For a robust statistical comparison of all 4-6h/day CR treated patients with the rest of the patients we pooled two big groups with comparable sizes: the "effective stimulation group" G1 + G3 $(n=34)$ was compared with the "ineffective stimulation group" comprising all data of G2 ("noisy" stimulation with a masker effect but without lasting off-effect), G4 (only $1 \mathrm{~h}$ of CR neuromodulation per day leading to smaller improvements) and G5 (the placebo group) with $n=29$ patients, see Table 4.

As a result, after 12 weeks of stimulation VAS loudness $(p=0.0009, p=0.0186)$, VAS annoyance $(p=0.0021, p=0.0488)$ and TQ $(p=0.0076)$ all showed markedly higher improvements in the "effective stimulation group" compared to the "ineffective stimulation group" (for on-, off-stimulation if applicable). Notably, the improvements in tinnitus severity and loudness were accompanied by a significant and pronounced shift in tinnitus frequency in the pooled CR neuromodulation group (between $-16.5 \%$ and $-22.2 \%$ average Tinnitus frequency shift in $\%$ of baseline) but not for the pooled "ineffective stimulation group" (between $+2.4 \%$ and $-2.6 \%$ average tinnitus frequency shift in $\%$ of baseline), see Table 4.

Fig. 2. Clinical effects of acoustic CR neuromodulation assessed in the framework of the RESET study. (a) Visual analogue scale (VAS) scores for loudness (left) and annoyance (right) for group 1 to 5. The VAS scores are displayed at baseline (0) and at 4, 12 and 16 weeks. The black (red) lines indicate the VAS scores obtained during off-stimulation (on-stimulation). The gray shaded area indicates the 12 weeks treatment phase, followed by a treatment pause of 4 weeks. The stars indicate significant results as compared to baseline. Acoustic CR neuromodulation induced a significant long-lasting reduction of VAS scores (off-stimulation, black) for therapy groups 1 and 3 while groups 2, 4 and 5 (placebo stimulation) showed no significant long-lasting treatment effects. Additionally, therapy groups 1 to 4 showed strong beneficial effects during stimulation (on-stimulation, red). (b) Changes of the VAS scores for loudness (left) and annoyance (right) with respect to baseline for the 12-week visit (left side of each plot) and the 16-week visit (right side of each plot) are given. The scores are plotted for off-stimulation (top) and on-stimulation (bottom) for G1 to G5 (from left to right). The stars indicate significant results as compared to placebo. Since at 16-week visit placebo (G5) started to receive G1 treatment, no data for on-stimulation was available. (c) On/Off-treatment effects $\left(\Delta V S_{\text {on }}-\Delta\right.$ VAS $\left.{ }_{\text {off }}\right)$ at 12 weeks are given. The stars indicate significant results as compared to placebo. (d) Tinnitus related distress level based on the tinnitus questionnaire (TQ, ranging from 0 to 84 points) is given (Goebel and Hiller 1998). Subjects are grouped into four severity levels: very severe (60-84, black), severe (47-59, dark gray), moderate (31-46, light gray) or mild (0-30, white). TQ severity level distribution is displayed at baseline, 12 and 16 weeks. Improvement in tinnitus severity level distribution was significant for G1 $(p<0.01)$, G3 and G4 ( $p<0.05)$, but not for placebo (G5) (stars indicate significant results as compared to baseline, Sign-nonparametric test, $p$-values (12/16 weeks): G1 0.0002/0.0003, G2:0.48/--, G3 : 0.023/0.041, G4 : 0.041/0.08, G5 : 0.48/_-; - - test not applicable due to no level variation). (e) Treatment induced changes in oscillatory EEG activity (off-stimulation). EEG activity for G1 (top) and placebo (bottom) was assessed at baseline (white) and at 12 weeks (gray). The normalized EEG power in the $\delta$ and $\alpha$ band is shown for G1 $(n=21$ evaluable) and G3 $(n=5)$. Stars indicate significant results as compared to baseline. (f) Correlation between $\triangle V_{\text {VAS }}$ Loudness and $\triangle \mathrm{VAS}_{\text {Annoyance. }} \Delta$ VAS-values are calculated for the 12-week visit (off-stimulation) compared to baseline. Changes of tinnitus loudness and annoyance are strongly and significantly correlated $(r=0.92, p<0.001)$. Dashed lines indicate $95 \%$ confidence levels. For all plots: mean values (a-c, e) and TQ grouping (d) is shown, ${ }^{*} p<0.05, * * p<0.01$. 
Table 2

Change from baseline to end of treatment phase (12 week follow up) and after the 4 weeks treatment pause (16 week follow up) in primary efficacy outcomes and EEG

\begin{tabular}{|c|c|c|c|c|c|c|c|c|c|c|}
\hline \multirow{2}{*}{$\begin{array}{l}\text { Treatment-group } \\
\text { Follow-up (week) }\end{array}$} & \multicolumn{2}{|c|}{ G1 $(n=22)$} & \multicolumn{2}{|c|}{$\mathrm{G} 2(n=12)$} & \multicolumn{2}{|c|}{ G3 $(n=12)$} & \multicolumn{2}{|c|}{ G4 $(n=12)$} & \multicolumn{2}{|c|}{ G5 $(n=5)$} \\
\hline & 12 & 16 & 12 & 16 & 12 & 16 & 12 & 16 & 12 & 16 \\
\hline \multicolumn{11}{|l|}{ VAS Loudness } \\
\hline $\begin{array}{l}\text { Change from baseline } \\
\text { (off-stimulation) [mean (SD)] }\end{array}$ & $-21.8(19.2)$ & $-17.0(22.8)$ & $-2.1(21.7)$ & $2.1(22.4)$ & $-25.8(25.3)$ & $-18.3(26.8)$ & $-6.7(15.3)$ & $-6.7(11.5)$ & $-9.0(18.8)$ & $-1.0(28.8)$ \\
\hline$p$ value (change from baseline $\S \S \S$ ) & $<0.0001$ & 0.0010 & 0.8438 & 0.8516 & 0.0039 & 0.0293 & 0.2969 & 0.0742 & 0.5000 & 0.8750 \\
\hline $\begin{array}{l}\text { Change from baseline } \\
\text { (on-stimulation) [mean (SD)] }\end{array}$ & $-37.3(24.7)$ & $-37.3(25.1)$ & $-21.3(25.3)$ & n.a. $\S \oint$ & $-29.6(30.0)$ & n.a. $\oint \oint$ & $-18.8(23.7)$ & $-22.1(27.2)$ & $-9.0(18.8)$ & n.a. $\oint \oint$ \\
\hline$p$ value (change from baseline $\S \S \S$ ) & $<0.0001$ & $<0.0001$ & 0.0195 & & 0.0078 & & 0.0254 & 0.0156 & 0.5000 & \\
\hline \multicolumn{11}{|l|}{ VAS Annoyance } \\
\hline $\begin{array}{l}\text { Change from baseline } \\
\text { (off-stimulation) [mean (SD)] }\end{array}$ & $-18.0(17.2)$ & $-11.4(24.2)$ & $-4.2(24.6)$ & $-5.0(22.3)$ & $-28.8(27.0)$ & $-20.4(26.7)$ & $-7.5(16.7)$ & $-5.0(18.5)$ & $-2.0(16.4)$ & $2.0(33.5)$ \\
\hline$p$ value (change from baseline $\oint \S \S$ ) & 0.0001 & 0.0381 & 0.6113 & 0.5830 & 0.0039 & 0.0215 & 0.2813 & 0.3828 & 1.0000 & 1.0000 \\
\hline $\begin{array}{l}\text { Change from baseline } \\
\text { (on-stimulation) [mean (SD)] }\end{array}$ & $-32.7(23.2)$ & $-33.2(22.8)$ & $-22.1(33.5)$ & n.a. $\oint \oint$ & $-31.7(33.3)$ & n.a. $\S \S$ & $-18.8(23.7)$ & $-21.3(28.1)$ & $-8.0(13.0)$ & n.a. $\oint \oint$ \\
\hline$p$ value (change from baseline $\S \S \S$ ) & $<0.0001$ & $<0.0001$ & 0.0566 & & 0.0103 & & 0.0195 & 0.0195 & 0.5000 & \\
\hline \multicolumn{11}{|l|}{ TQ score } \\
\hline $\begin{array}{l}\text { Change from baseline } \\
\text { (off-stimulation) [mean (SD)] }\end{array}$ & $-12.4(8.9)$ & $-12.3(10.9)$ & $-5.2(8.0)$ & $-6.2(8.1)$ & $-15.5(15.1)$ & $-17.7(17.5)$ & $-8.6(7.0)$ & $-10.5(10.6)$ & $-8.4(7.1)$ & $-9.2(10.5)$ \\
\hline$p$ value (change from baseline ${ }^{\complement} \S$ ) & $<0.0001$ & $<0.0001$ & 0.0273 & 0.0166 & 0.0068 & 0.0024 & 0.0029 & 0.0068 & 0.1250 & 0.2500 \\
\hline \multicolumn{11}{|l|}{ Tinnitus frequency } \\
\hline (off-stimulation) [mean \% (SD)] & & & & & (10.0) & & & & & \\
\hline $\begin{array}{l}p \text { value (change from baseline }{ }^{\S \S} \S \text { ) } \\
\text { EEG } \delta \text { power }\end{array}$ & 0.0001 & $<0.0001$ & 0.5693 & 0.3804 & 0.0640 & 0.0425 & 0.5186 & 0.8984 & 0.0625 & 0.0625 \\
\hline $\begin{array}{l}\text { Change from baseline } \\
\text { (off-stimulation) [mean (SD)] }\end{array}$ & $-0.065^{\S}(0.084)$ & & $-0.002^{\S}(0.021)$ & & $-0.071(0.032)$ & & $-0.0008(0.009)$ & & $0.0002(0.07)$ & \\
\hline$p$ value (change from baseline $\S \S \S$ ) & 0.0007 & & 0.2500 & & 0.0170 & & 0.2750 & & 0.89 & \\
\hline EEG $\alpha$ power & & & & & & & & & & \\
\hline $\begin{array}{l}\text { Change from baseline } \\
\text { (off-stimulation) [mean (SD)] }\end{array}$ & $0.11^{\S}(0.12)$ & & $0.009^{\S}(0.033)$ & & $0.13(0.09)$ & & $0.007(0.028)$ & & $-0.0095(0.1)$ & \\
\hline$p$ value (change from baseline $\S \S \S$ ) & 0.0007 & & 0.3400 & & 0.0030 & & 0.2500 & & 0.89 & \\
\hline
\end{tabular}

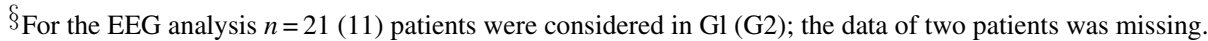

$\S \S 16$-week measurements of VAS Loudness and Annoyance in the on-stimulation condition were done for Gl and G4 only. Therefore, for G2, G3 and G5 this test was not applicable (n.a.).

$\S \S \S$ Wilcoxon matched pairs test. 


\subsubsection{Comparison to masker type of stimulation (noisy $C R, G 2$ )}

All groups were compared with the "noisy" masker like stimulation (G2) that showed a good on-effect like G1 and G3 but no lasting off-effect. After 12 weeks of treatment significant differences occurred for TQ in favor of G1 $(p=0.0171)$ and G3 $(p=0.0347)$, for VAS loudness off-stimulation in favor of G1 $(p=0.0166)$ and G3 $(p=0.0330)$ and for VAS annoyance offstimulation in favor of G3 $(p=0.0233)$.

\subsubsection{EEG analysis}

Source montage analysis of whole-head EEG measurements revealed a significant decrease of $\delta$ band power and increase of $\alpha$ band power in the primary auditory cortex of G1 $(p<0.01)$ while no significant changes in G5 (placebo) were observed ( $p=0.89$, Fig. 2e, Table 2). Additionally, we determined 3D maps of significant changes of oscillatory brain activity, using the sLORETA technique, in typical frequency bands in 12 patients with bilateral tinnitus taken from the stimulation groups G1-G4 (see Methods). Reduced levels of $\alpha$ band power were significantly increased after 12 weeks of treatment (red voxels, $p<0.05$, Fig. 3): the widespread bilateral $\alpha$ increase was strongest in temporal regions and the entire prefrontal cortex (Fig. 3 and Table 3). Tinnitus-associated enhanced $\delta$ and $\gamma$ activity was significantly reduced in a widespread network comprising auditory and non-auditory areas (blue voxels, $p<0.05$, Fig. 3 ): $\delta$ activity was decreased mainly in temporal and prefrontal regions including the primary and secondary auditory cortices. $\theta$ activity was significantly decreased in frontal regions and the anterior cingulate area (Brodmann Area (BA) 32). A significant decrease in $\beta$ activity was observed in temporal areas, therein maximal in the superior temporal gyrus (bilateral, BA 41, 42). $\gamma_{\text {low }}$ activity, i.e. $\gamma$ activity of low frequency (see Methods), was significantly decreased in the temporal and frontal cortex covering wider areas in the right prefrontal cortex, while $\gamma_{\text {high }}$ showed a left-centered significant decrease in the temporal cortex (maximal in the superior temporal gyrus, BA 41). All results are displayed in Fig. 3 (red voxels, $p<0.05)$, corresponding Brodmann areas are listed in Table 3 .

\subsubsection{Long-term extension}

In LTE (all subjects receiving CR neuromodulation like in G1; 58 subjects started, 52 subjects completed LTE) already gained treatment effects were sustained or even improved further. The overall improvement in TQ was $36 \%(p<0.0001 t$-test, compared to baseline). Using the responder analysis by Goebel et al. (2006) we found at the end of 40 weeks $40 \%$ winners (TQ improvement $\geq 15$ points), $35 \%$ responders (TQ improvement 6-14 points), 23\% non-responders (TQ unchanged \pm 5 points) and $2 \%$ losers (TQ worsening $\geq 6$ points).

\subsubsection{Safety}

15 AEs occurred in total: 13 AEs during blinded phase, 2 AEs in LTE. Two SAEs (an abdominal pregnancy and avascular necrosis of the femoral head, not associated with treatment) were reported. All other AEs were of mild to moderate intensity and none was permanent. 8 AEs were judged to be treatment related of which 3 AEs were associated with a transient increase of tinnitus loudness; all three patients continued treatment into the LTE.

\section{Discussion}

\subsection{Clinical effects}

We showed that acoustic CR neuromodulation was safe and well-tolerated. We found a significant and clinically relevant concordant strong decrease of VAS scores (loudness and annoyance), TQ scores and TQ severity levels. Improvements persisted a preplanned 4-week therapy pause on a slightly reduced, but significant level, regaining the reduction in VAS after continuation of the CR therapy and showing sustained long-term effects during the LTE. At the end of LTE (at 40 weeks) we observed $75 \%$ winners and responders with a mean TQ reduction of 50\%. A similar reduction of tinnitus distress, i.e. $70 \%$ of winners/responders in TQ after 6 months of acoustic CR neuromodulation, was shown in a cohort of seventy "real life" patients suffering from chronic tonal tinnitus in an outpatient setting (TRI Tinnitus Conference 2011, Buffalo, USA, Abstract H. Wurzer). In contrast, when treated with 

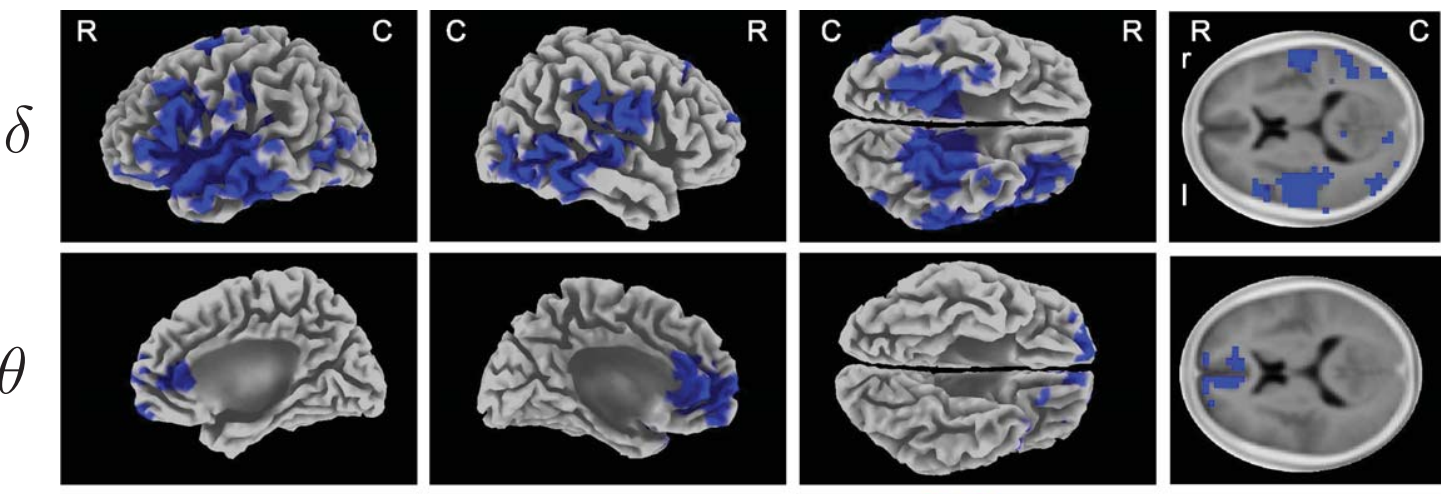

$\theta$
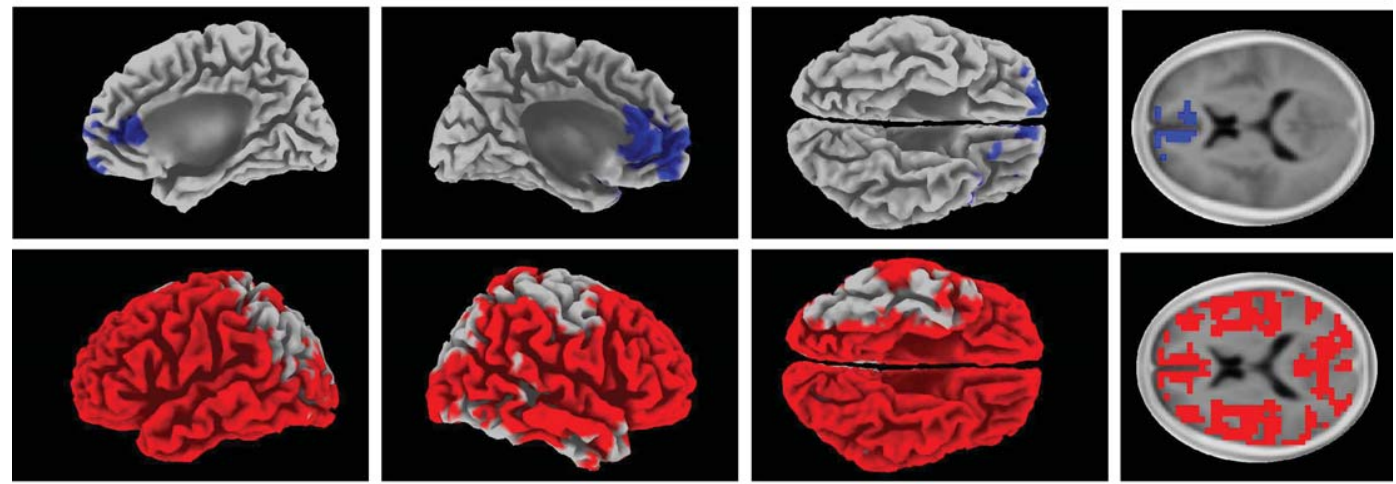

$Q$
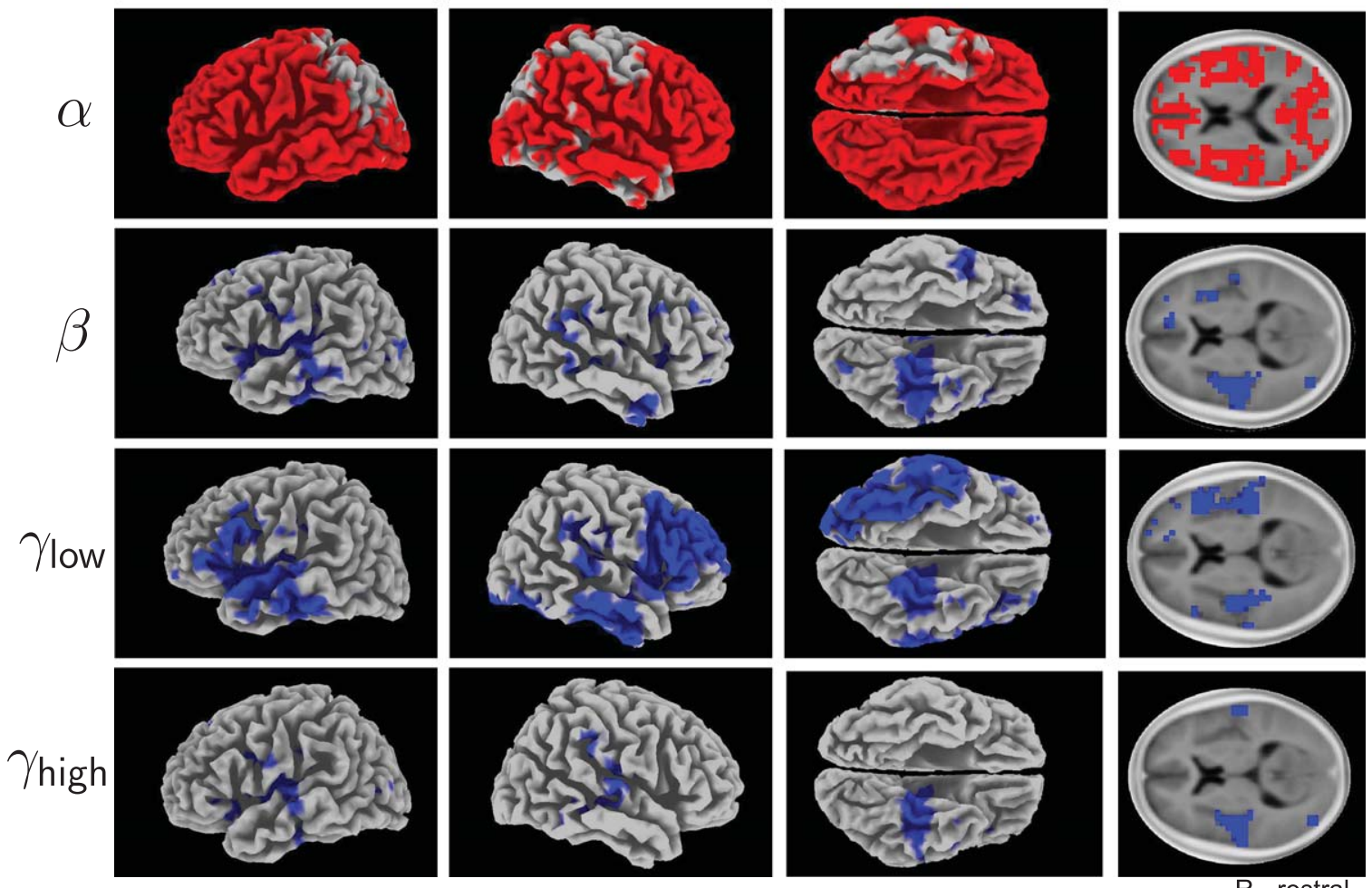

R - rostral

differences in neuronal activity (sLORETA)

C - cauda

$r$ - right

I - left

Fig. 3. Electrophysiological effects of acoustic CR neuromodulation assessed in the framework of the RESET study. 3D mapping of treatment induced changes in oscillatory EEG activity (baseline compared to 12 weeks, off-stimulation). To increase signal-to-noise ratio 12 patients with bilateral tinnitus (from G1 to G4) were selected using reliable-change-index (RCI) applied to improvements of TQ scores. Statistical non-parametric maps from sLORETA (Pascual-Marqui, 2002) for the localization of changes of $\delta, \theta, \alpha, \beta, \gamma_{\text {low }}$ and $\gamma_{\text {high }}$ current source density are given. Results are superimposed onto a three-dimensional brain (first three columns) and onto a horizontal brain section (right column) of a standard anatomical template. Significantly decreased activity after acoustic CR neuromodulation compared to baseline is labeled blue, increased activity is labeled red (corrected, $p<0.05$ ).

noisers and maskers only a minority of patients experiences tinnitus-suppressing after-effects, and these last only seconds to minutes (Surr et al., 1985; Roberts et al., 2010). Comparing the active arms, CR therapy was more efficacious when used 4-6h/d compared to $1 \mathrm{~h} / \mathrm{d}$, and the results of noisy CR neuromodulation (G2) were less efficacious and importantly lacked a lasting off-stimulation effect resembling the typical short 
Table 3

Significant effects of acoustic CR neuromodulation on the EEG activity (baseline compared to 12 week follow up). The Brodmann areas where significant changes were observed are listed. Arrows indicate the effect of the treatment $(\downarrow:$ decrease, $\uparrow:$ increase, corrected, $p<0.05)$. Changes were localized using statistical non-parametric maps from sLORETA (Pascual-Marqui, 2002)

\begin{tabular}{|c|c|c|c|c|c|}
\hline \multirow[t]{2}{*}{ Band } & \multirow{2}{*}{$\begin{array}{l}\text { Frequency } \\
\text { range }\end{array}$} & \multirow[t]{2}{*}{ Effect } & \multicolumn{3}{|c|}{ Position } \\
\hline & & & Left & Bilateral & Right \\
\hline$\delta$ & $1-4 \mathrm{~Hz}$ & & $\begin{array}{l}\text { Prefrontal 46, } 47 \\
\text { Orbilo frontal } 11 \\
\text { Frontal } 44\end{array}$ & $\begin{array}{l}\text { Postcentral 1, 2; Precentral } 4 \\
\text { Prefrontal 6, 8, 9, 10; Insula } 13 \\
\text { Occipital 18, 19; Fusiform } 20 \\
\text { Temporal 21, 22, 28, 37, 38, 39, 41, } 42 \\
\text { Cingulate cortex 23, 31, 29 } \\
\text { Parahippocampus 30, 36 } \\
\text { Subcentral 43; Frontal } 45\end{array}$ & Parietal 40 \\
\hline$\theta$ & $4-8 \mathrm{~Hz}$ & $\downarrow$ & $\begin{array}{l}\text { Prefrontal } 47 \\
\text { Temporal } 38\end{array}$ & $\begin{array}{l}\text { Prefrontal 9, 10; Orbitofrontal } 11 \\
\text { Anterior cingulate } 32\end{array}$ & \\
\hline$\alpha$ & $8-12 \mathrm{~Hz}$ & $\uparrow$ & & $\begin{array}{l}\text { Postcentral 1, 2, 3; Precentral } 4 \\
\text { Prefrontal 6, 8, 9, 10, 46; Parietal 7; } \\
\text { Orbitofrontal 11, 47; Insula } 13 \\
\text { Occipital 17, 18, 19; Fusiform } 20 \\
\text { Temporal 21, 22, 28, 34, 35, 37, 38, 39, 41, } 42 \\
\text { Posterior cingulate 23, } 31 \\
\text { Anterior cingulate 24, 32, } 33 \\
\text { Frontal 25, 44, 45; Piriform } 27 \\
\text { Cingulate cortex 29; Parahippocampus 30, } 36 \\
\text { Parietal 40; Subcentral } 43\end{array}$ & \\
\hline$\beta$ & $12-30 \mathrm{~Hz}$ & $\downarrow$ & $\begin{array}{l}\text { Parietal } 39 \\
\text { Temporal } 37 \\
\text { Occipital } 18,19\end{array}$ & $\begin{array}{l}\text { Postcentral 2; Parietal } 40 \\
\text { Frontal 47; Fusiform } 20 \\
\text { Temporal 21, 22, 41, } 42\end{array}$ & $\begin{array}{l}\text { Prefrontal } 10 \\
\text { Orbitofrontal } 11\end{array}$ \\
\hline$\gamma_{\text {low }}$ & $30-48 \mathrm{~Hz}$ & $\downarrow$ & $\begin{array}{l}\text { Piriform } 27 \\
\text { Parahimpocampus } \\
30\end{array}$ & $\begin{array}{l}\text { Postcentral 1, 2; Precentral } 4 \\
\text { Prefrontal 6, 8, 9, 10, 46, 47; Orbitofrontal } 11 \\
\text { Insula 13; Fusiform } 20 \\
\text { Temporal 21, 22, 35, 37, } 41 \\
\text { Parahimpocampus 36; Frontal 44, 45, } 47\end{array}$ & $\begin{array}{l}\text { Occipital } 17,18 \\
\text { Temporal } 28 \\
\text { Parietal } 40\end{array}$ \\
\hline$\gamma_{\text {high }}$ & $52-90 \mathrm{~Hz}$ & $\downarrow$ & $\begin{array}{l}\text { Precentral } 4 \\
\text { Prefrontal } 9 \\
\text { Frontal } 45,47 \\
\text { Occipital } 19\end{array}$ & $\begin{array}{l}\text { Insula } 13 \\
\text { Temporal } 21,22,41,42 \\
\text { Subcentral 43, } 44\end{array}$ & $\begin{array}{l}\text { Postcentral } 1,2 \\
\text { Temporal } 20 \\
\text { Parietal } 40\end{array}$ \\
\hline
\end{tabular}

term effects of noisers or maskers (Terry et al., 1983). In contrast, placebo treatment showed limited and nonsignificant changes for VAS scores and TQ, which were in the range of previously reported effects of inpatient treatment including cognitive behavioral therapy (CBT, Goebel et al., 2006). This could be indicative of a "true" placebo effect in our study; oftentimes instead of placebo groups "waiting list control groups" are used that sometimes improve slightly on tinnitus specific measurements (Hesser et al., 2011) but studies have also shown no change or even worsening of the waiting list control group over time (Caffier et al., 2006).

The placebo group tended to be older and had a longer lasting tinnitus with lower VAS and TQ scores at baseline. However, tinnitus patients with a longer tinnitus duration ( $>4$ years) benefited in the same order of magnitude as patients with shorter tinnitus duration ( $<4$ years) indicating that $\mathrm{CR}$ effect is independent of tinnitus duration. The cutoff of 4 years was chosen, since MEG studies (Schlee et al., 2009b) and EEG studies (Vanneste et al., 2011) indicate a differential pattern of cerebral activity and connectivity in patients with shorter tinnitus duration ( $<4$ years) as opposed to longer tinnitus duration ( $>4$ years). This seems to differentiate acoustic CR Neuromodulation from repetitive transcranial magnetic stimulation (rTMS) and neurofeedback training for tinnitus where studies showed a reduction of treatment effects with increasing tinnitus duration (Langguth et al., 2003; Frank et al., 2010; Schlee et al., 2009b; Dohrmann et al., 2007a). Additionally, no correlation was found between age and treatment effect analyzing 12-week treatment of 
Table 4

Comparison of the pooled groups G1 \& G3 ( $n=34$, with 4-6hours/day acoustic CR Neuromodulation, "effective treatment groups") and G2, G4 \& G5 ( $n=29$, "ineffective treatment groups"). The change from baseline to end of treatment phase (12 week follow up) and after the 4 weeks treatment pause (16 week follow up) in primary efficacy outcomes

\begin{tabular}{|c|c|c|c|c|}
\hline \multirow{2}{*}{$\begin{array}{l}\text { Pooled-groups } \\
\text { Follow-up (week) }\end{array}$} & \multicolumn{2}{|c|}{ G1 \& G3 $(n=34)$} & \multicolumn{2}{|c|}{$\mathrm{G} 2, \mathrm{G} 4 \& \mathrm{G} 5(n=29)$} \\
\hline & 12 & 16 & 12 & 16 \\
\hline \multicolumn{5}{|l|}{ VAS Loudness } \\
\hline Change from baseline (off-stimulation) [mean (SD)] & $-23.2(21.2)$ & $-17.5(23.9)$ & $-5.2(18.3)$ & $-2.1(19.6)$ \\
\hline$p$ value (change from baseline ${ }^{\# \#}$ ) & $<0.0001$ & 0.0003 & 0.1752 & 0.5677 \\
\hline Change from baseline (on-stimulation) [mean (SD)] & $-34.6(26.5)$ & n.a. & $-18.1(23.3)$ & n.a. ${ }^{\#}$ \\
\hline$p$ value (change from baseline ${ }^{\# \#}$ ) & $<0.0001$ & & 0.0008 & \\
\hline \multicolumn{5}{|l|}{ VAS Annoyance } \\
\hline Change from baseline (off-stimulation) [mean (SD)] & $-21.8(21.4)$ & $-14.6(25.1)$ & $-5.2(19.8)$ & $-3.8(22.3)$ \\
\hline$p$ value (change from baseline ${ }^{\# \#}$ ) & $<0.0001$ & 0.0027 & 0.2513 & 0.3986 \\
\hline Change from baseline (on-stimulation) [mean (SD)] & $-32.4(26.7)$ & n.a. ${ }^{\#}$ & $-18.3(26.7)$ & n.a. ${ }^{\#}$ \\
\hline$p$ value (change from baseline ${ }^{\# \#}$ ) & $<0.0001$ & & 0.0018 & \\
\hline \multicolumn{5}{|l|}{ TQ score } \\
\hline Change from baseline (off-stimulation) [mean (SD)] & $-13.5(11.3)$ & $-14.2(13.6)$ & $-7.1(7.3)$ & $-8.5(9.5)$ \\
\hline$p$ value (change from baseline $\mathrm{e}^{\# \#}$ ) & $<0.0001$ & $<0.0001$ & $<0.0001$ & 0.0002 \\
\hline \multicolumn{5}{|l|}{ Tinnitus frequency } \\
\hline Relative change from baseline (off-stimulation) [mean \% (SD)] & $-22.2(35.5)$ & $-16.5(62.3)$ & $2.4(36.9)$ & $-2.6(35.8)$ \\
\hline$p$ value (change from baseline $\mathrm{e}^{\# \#}$ ) & $<0.0001$ & $<0.0001$ & 0.5377 & 0.3389 \\
\hline
\end{tabular}

\# 16-week measurements of VAS Loudness and Annoyance in the on-stimulation condition were done for G1 and G4 only. Therefore, for G2, G3 and G5 this test was not applicable (n.a.).

\#\#Wilcoxon matched pairs test.

all 4-6 h/day CR treated patients and analyzing of all patients after 40 weeks of treatment with CR.

Furthermore, using the classification by (Goebel and Hiller, 1998), we observed that the relative change in TQ score, i.e. the differences compared to baseline, was in the same order for more severely impaired (so called "decompensated" tinnitus, TQ $>46$ ) as for less severely impaired patients (so called "compensated" tinnitus, TQ $\leq 46$ ). Analyzing for tinnitus severity in the 12 weeks and 40 weeks $4-6 \mathrm{~h} /$ day CR-treated patients likewise did not reveal baseline TQ score as confounding factor for tinnitus success (see result section).

Remarkably, reduction of tinnitus loudness and reduction of tinnitus annoyance, as measured with VAS, were highly correlated in the RESET trial. This is in line with Tyler et al. (2007) who were able to show a correlation between loudness and annoyance. Furthermore, we found a robust correlation between the reduction of tinnitus loudness and reduction of TQ scores (relative values, $r=0.43, p<0.001$ ).

The pronounced and sustained reduction of the subjective loudness of tinnitus is an effect of acoustic $\mathrm{CR}$ neuromodulation which has not been observed for instance with cognitive behavioural therapy (CBT) (Martinez-Devesa et al., 2010; Goebel et al., 2006). CR therapy significantly decreased tinnitus frequency whereas placebo did not, indicative of neuroplastic changes induced by $\mathrm{CR}$ neuromodulation.

\subsection{EEG changes}

After 12 weeks of acoustic CR neuromodulation pathologically elevated $\delta$ and $\gamma$ activity were both decreased in primary and secondary auditory cortex as well as in frontal brain areas. Concomitantly, tinnitus related reduction of $\alpha$ activity was reversed and enhanced $\alpha$ activity reoccurred in auditory and prefrontal areas.

An altered pattern of ongoing oscillatory activity in the auditory cortex represents the underlying neural code of tinnitus. Enhancements in the $\gamma$ frequency band, which can be assumed to be a sign of enhanced synchronized firing of neurons, are involved in the formation of phantom perceptions such as tinnitus (Weisz et al., 2007). Attempts to modify ongoing spontaneous oscillatory activity and measure changes in the perception or, manipulate the tinnitus and observe concomitant changes in brain activity can both be successful as tinnitus therapy. The first strategy was tested using neurofeedback normalizing the spontaneous activity pattern by enhancing $\alpha$ power and reducing $\delta$ power. Concomitant changes in both bands lead to the greatest reductions of tinnitus loud- 
ness (Dohrmann et al., 2007b). The other strategy of reducing the intensity of the tinnitus and to observe whether concomitant changes occur in the electrophysiological activity was carried out in this study with acoustic CR neuromodulation.

The EEG pattern of our patients prior to treatment resembles previous findings by Weisz et al. (2005a) who showed a marked reduction of $\alpha$ along with an enhancement of $\delta$ frequency power in tinnitus patients particularly in temporal regions. In our study we additionally found $\alpha$ reduction and $\delta$ enhancement in associative and limbic areas (prefrontal and cingulate). Functional connectivity of these areas has been described as basis for impairment and many secondary tinnitus symptoms (Llinás et al., 1999; Tyler, 2006; Schlee et al., 2009b; Moazami-Goudarzi et al., 2010; Rauschecker et al., 2010). These findings are in line with the notion of Llinás et al. (1999) and Jeanmonod et al. (1996), that clinical symptoms, e.g., neurogenic pain, tinnitus, abnormal movements, epilepsy and certain neuropsychiatric disorders, are associated with low frequency rhythmic bursting activity.

Neuromagnetic changes caused by maskers in chronic tinnitus sufferers (Kahlbrock and Weisz, 2008) showed a decrease of slow-wave spontaneous brain activity from pre- to post-stimulation caused by residual inhibition (RI), i.e. a suppression of pathologic neuronal activity. The corresponding suppression of tinnitus extends only seconds or minutes beyond the duration of the direct stimulation. In contrast, acoustic $\mathrm{CR}$ neuromodulation decreases the slow-wave $(\delta)$ activity by desynchronization leading to long-lasting therapy induced changes of spontaneous EEG activity after 12 weeks of therapy measured in the off-state, namely a $\delta$ reduction and $\alpha$ enhancement, indicative of neuroplastic changes by acoustic neuromodulation, modifying the neuronal correlates of tinnitus in these areas in our patients (Weisz et al., 2005a; Dohrmann et al., 2007a). So we here focus the outcome measures on assessing the impact of the treatment based on the neurophysiological pathway of tinnitus as suggested by the Cochrane collaboration (Martinez-Devesa et al., 2010).

\subsection{Long-lasting therapy induced changes}

In our study the decrease of tinnitus frequency, EEG changes and persistence of clinical improvement during the 4-week therapy pause (instead of complete wash-out) might be indicative of neuroplastic changes.
In computational studies it was shown that CR neuromodulation induces a long-lasting desynchronization mediated by an unlearning of synaptic connectivity (Tass and Majtanik, 2006; Hauptmann and Tass, 2007; Tass and Hauptmann, 2009; Tass and Popovych, 2012). The EEG changes and the persistence of clinical improvement during the 4-week therapy pause observed in our study are in accordance to the predictions of these computational studies. However, given the results presented here, we cannot prove that in the tinnitus patients CR works as shown computationally, i.e. by means of a sequential phase reset of neuronal sub-populations (Tass, 2003). Accordingly, in a forthcoming EEG study we shall investigate whether and, if so, in which cortical areas single tones induce a phase reset of pathological rhythms, whereas CR stimuli (i.e. time-shifted sequences of phase resetting tones) cause a desynchronization (i.e. a decrease of the power in pathological frequency bands).

This forthcoming study might be helpful to further reveal the mechanism underlying our therapy.

Based on the data presented here, we cannot rule out that a qualitatively different mode of action might lead to the observed long-lasting clinical and electrophysiological changes.

In fact, the acoustic stimulation delivered to our patients might have primarily changed the synaptic connectivity which, in turn, might have caused a decrease of neuronal synchronization. Also, the mechanism underlying residual inhibition (RI, Feldmann, 1971; Hazell and Wood, 1981; Terry et al., 1983) is not yet understood. Long-lasting RI, observed e.g. after 15min masking periods, occurs only in a small percentage of patients (Hazell and Wood, 1981). Accordingly, the significant off-stimulation effects outlasting the treatment pause of four weeks which were observed in G1 can hardly be explained by a RI-related mechanism. Mechanisms primarily employing lateral inhibition and, hence, a suppression of the tinnitus-related neuronal synchronization cannot be ruled out based on the results presented here. However, such a mechanism would be in contradiction to computational results showing that blocking/inhibitory stimulation cannot induce an unlearning of synaptic connectivity, since the latter requires neurons to be active (Tass and Majtanik, 2006; Hauptmann and Tass, 2007; Tass and Hauptmann, 2009; Tass and Popovych, 2012). A phenomenon that might be related to this issue is well-known from electrical deep brain stimulation in Parkinson's disease (PD). High-frequency electrical 
deep brain stimulation (Benabid et al., 1991) has no long-lasting clinical effects: PD symptoms reappear after cessation of stimulation (Temperli et al., 2003). Analogously, PD-related oscillatory $\beta$ band activity, suppressed during stimulation, reemerges after cessation of stimulation within seconds (Kühn et al., 2008).

Another remarkable phenomenon is the tinnitus pitch change. This phenomenon has not been predicted by our computational studies. In fact, the underlying physiological mechanism of the CR-induced tinnitus pitch change still remains open. Taking into account the tonotopic organization of the primary auditory cortex, a CR-induced tinnitus pitch change may likely correspond to a spatial shift of the tinnitus-related focus of synchronized neuronal activity within the primary auditory cortex. Based on the dynamical mode of action of CR neuromodulation (Tass, 2003; Tass and Majtanik, 2006; Hauptmann et al., 2007; Tass and Hauptmann, 2009; Tass and Popovych, 2012) such an effect might be caused by asymmetries of both the stimulation and the network subjected to stimulation: (i) In a modeling study (Franosch et al., 2003) on the emergence of the Zwicker tone (Zwicker, 1964), i.e. an auditory after effect, the impact of a spatial gradient of the lateral inhibition in the auditory cortex on the neuronal dynamics in the central auditory cortex has been analyzed. It turned out that a gradient of the lateral inhibition has significant impact on the neuronal dynamics. In particular, a noise reduction mechanism combined with a dominantly unilateral inhibition is able to explain the emergence of the Zwicker tone (Franosch et al., 2003). Such a gradient of the lateral inhibition combined with a spatially equidistant delivery of acoustic stimuli might be a candidate mechanism causing a spatial shift of the synchronous focus in the primary auditory cortex. (ii) In case of a spatially well-balanced lateral inhibition asymmetries of the alignment of the CR tones (with respect to the patient's individual tonotopic organization of the primary auditory cortex) might also spatially shift the synchronous focus in the primary auditory cortex. Possible mechanisms underlying the shift of the tinnitus frequency will be in the focus of forthcoming theoretical and experimental studies.

\subsection{Comparison with other therapeutic approaches}

Vagus nerve stimulation (VNS) combined with multiple tone pairing - an invasive therapy tested so far in animals - aims at counteracting tinnitus by controlling map plasticity (Engineer et al., 2011).

Another invasive approach to suppress synchronized hyperactivity associated with tinnitus has been carried out by De Ridder and his group (De Ridder et al., 2007): after a complex diagnostic procedure with fMRI and neuronavigation-guided trans-magnetic stimulation (TMS) patients responding to TMS received via craniotomy the insertion of an epidurally fixed octopolar electrode and consequent bipolar stimulation by means of a pulse generator. A reduction of tinnitus loudness measured by a VAS scale occurred in tonal tinnitus whereas in atonal tinnitus the technique failed to show significant results. After a while, tinnitus reoccurred in all patients and reorganization of the electrode array was necessary (De Ridder et al., 2006).

Based on initial animal studies tinnitus is proposed to be an auditory phantom phenomenon associated with a synchronized hyperactivity and reorganization of the two auditory pathways (lemniscal and extralemniscal) all the way up to the auditory cortex (Moller, 2007).

The correlation between tinnitus strength and the amount of reorganization of the primary cortex found in earlier study (Mühlnickel et al., 1998) was not replicated or weakened in subsequent studies (Weisz et al., 2005a; Yang et al., 2011).

In a previous study tailor-made notched music (i.e. music with a frequency band of one octave width centered at the individual tinnitus frequency removed from the music energy spectrum via digital notch filter) was used to counteract tinnitus-related auditory cortex reorganization. The presumed mechanism here is inhibition of tinnitus-related activity mediated by lateral inhibition (Okamoto et al., 2010). For the "placebo" group a moving notch filter of one octave around the tinnitus frequency was applied. A third group didn't receive any music treatment at all and served as a control group.

In this study patients with very mild to mild tinnitus distress were enrolled (TQ 18.4 \pm 10.8 ; scale 0-84). Both, the active group $(n=8)$ listening to tailor-made notched music and the control group $(n=7)$ without any music treatment showed after 6 months improvements in loudness (measured by a VAS scale, 0-100). On the other hand, the "placebo" group $(n=8)$ using a moving notch filter showed worsening of the perception of tinnitus loudness which might be explained by a tinnitus worsening effect of the moving notch filter technique itself. This observed deterioration of the 
placebo group contrasts with the improvements of the placebo group described here in RESET and contradicts also latest pharmacological studies in the field of tinnitus like the Neramexane trial (Suckfüll et al., 2011) where also a pronounced placebo effect was notable.

In contrast, the CR approach strictly avoids inhibition of pathologically synchronized activity (Tass and Majtanik, 2006; Hauptmann and Tass, 2007; Tass and Hauptmann, 2009). As shown computationally, in general, inhibitory stimulation is unfavorable for antikindling, since inactive neurons are unable to unlearn (Tass and Majtanik, 2006; Hauptmann and Tass, 2007; Tass and Hauptmann, 2009). However, CR robustly works even if only inhibitory synapses are stimulated (Hauptmann and Tass, 2007; Tass and Hauptmann, 2009), because a phase reset can be achieved by both excitatory and inhibitory stimuli (Winfree, 1977; Guttman et al., 1980).

Optimal desynchronizing CR effects require mutually sufficiently distant stimulation sites (here frequencies) (Tass, 2003; Lysyansky et al., 2011). As yet, there is no neuroimaging or electrophysiological technique available which enables to assess the spatial extent of the synchronized focus in tinnitus patients. Psychoacoustic measurements of tinnitus spectra revealed that broad frequency ranges, largely corresponding to ranges with abnormally elevated hearing thresholds, contribute to the patients' tinnitus sensations (Norena et al., 2002). The corresponding central auditory neurons, deprived of afferent input, were supposed to be engaged in the pathological, tinnitus-related neuronal activity (Norena et al., 2002). Accordingly, based on results from theoretical studies (Tass, 2003; Tass and Hauptmann, 2009; Lysyansky et al., 2011) we derived rules for choosing pitch and amplitude of the desynchronizing CR tones: Ideally the CR stimuli should be confined to the synchronized focus. However, CR is still (but less) effective if one or two stimulation sites are misplaced, e.g. because they are located outside the synchronized focus. Most importantly, a dense spacing of the stimulation sites places the desynchronizing effect in jeopardy. In fact, for sufficiently dense spacing of the stimulation sites the effect of CR stimulation finally approaches the synchronizing effect of a spatially homogenous stimulation that is periodic in time (Tass and Hauptmann, 2009). Accordingly, given the spatial profile of the tuning curves, especially in patients with impaired hearing, a spatially selective stimulation requires the
CR tones to be sufficiently spaced, equally loud and just super-threshold (see Methods). Accordingly, the masking effect in G2 suggests that due to a relevant portion of stimulation cycles with neighboring tones the noisy $\mathrm{CR}$ in $\mathrm{G} 2$ suppresses the tinnitus-related neuronal activity via lateral inhibition. The significant difference of the long-lasting, cumulative and longterm effects in G1/G3 vs. the masking effects in G2 indicate that the therapeutic outcome might be optimized by further adapting the arrangement of the CR frequencies to the individual tonotopy and the dimensions of the pathological synchronized activity in the primary auditory cortex. Also, a closer meshed adaptation of the repetition rate $F$ to the intrinsically varying $\delta$ peak frequency might possibly improve the therapeutic outcome.

\subsection{Neuronal synchronization}

Tinnitus animal research mainly focuses on neural correlates of short-term tinnitus. In several studies it was shown that noise trauma causes an immediate increase of neuronal cross-correlation, which may even precede the increase of the firing rates (Ochi and Eggermont, 1997; Norena and Eggermont, 2003; Seki and Eggermont, 2003). For several reasons comparison to results obtained in humans with MEG, EEG or LFP measurements has to be drawn carefully:

(i) Human EEG, MEG and LFP studies have typically been performed in patients with chronic tinnitus (see Weisz et al., 2005a; Weisz et al., 2007). In analogy to animal studies, in one human study a rapid increase of neuronal synchronization, in terms of a rapid increase of $\gamma$ power in the auditory cortex, following noise trauma was observed (Ortmann et al., 2011).

(ii) In tinnitus animal experiments and in human studies neuronal dynamics is studied on different scales. While in tinnitus animal studies typically neuronal spikes or bursts are analyzed (Eggermont, 1992; Ochi and Eggermont, 1997), in human studies LFP, scalp EEG and MEG oscillations are investigated, which are considered to be generated by oscillations of neocortical postsynaptic potentials (Klaas and Daly, 1979; Nunez, 1981; Hämäläinen et al., 1993; Niedermeyer and Lopes da Silva, 1999).

(iii) In this paper the term "neuronal synchronization" stands for coincident firing in a large 
population of neurons. This type of synchronization leads to large-amplitude oscillations as detected by LFP, EEG, and MEG signals, typically in particular frequency ranges (see Klaas and Daly, 1979; Nunez, 1981; Hämäläinen et al., 1993; Niedermeyer and Lopes da Silva, 1999). In contrast, cross correlation analysis is typically performed for single unit and/or multi unit data (Eggermont, 1992; Ochi and Eggermont, 1997). Qualitatively different types of collective synchronization patterns may lead to large-amplitude LFP, EEG and MEG oscillations, see e.g. (Popovych and Tass, 2011). However, fundamental dynamical phenomena, such as collective coincident firing, can be detected with different synchronization and cross correlation measures, for review see (Quiroga et al., 2002).

\subsection{Mechanisms of CR neuromodulation at the cortical level}

The auditory pathway has a complex, hierarchical tonotopic organization (Ehret and Romand, 1997). Based on the results presented here, we can hypothesize about how acoustic CR neuromodulation actually works at the cortical level. Based on previous, in particular computational studies, CR might cause a desynchronization of cortical neuronal populations in qualitatively different ways:

(i) Stimuli can effectively induce a phase reset no matter whether they act on neurons directly (as e.g., electrical pulses delivered to the neuronal membrane) or indirectly (i.e. transmitted via synapses) (Popovych and Tass, 2012; Tass and Popovych, 2012). Due to the tonotopic organization of the central auditory system, these phase resets may, hence, occur at different sites. The spatial spread of the different stimuli crucially depends on the characteristics of the tuning curves of auditory nerve fibers, which may, e.g., be pathologically broadened in patients with cochlear hearing loss (Wightman et al., 1977; Ryan et al., 1979; Liberman and Dodds 1984; Pickles, 1984). However, it has computationally been shown that CR neuromodulation is quite robust with respect to variations of the spatial spread (Lysyansky et al., 2011). So, in principle, acoustic CR neuromodulation might induce a desynchronization of the $\delta$ rhythm at the cortical level by inducing phase resets of the $\delta$ rhythm in different cortical subpopulations at different times. For a discussion of phase resets of ongoing brain rhythms we refer to (Klimesch et al., 2006).

(ii) However, desynchronization at the cortical level might also be induced by $\mathrm{CR}$ in a qualitatively different way. The typical CR mechanism, desynchronization via time-shifted phase resets of neuronal subpopulations (Tass, 2003), might take place at an upstream nucleus in the central auditory system. From computational studies it is known, that desynchronizing effects propagate between neuronal populations: This has been shown for qualitatively different (single-site vs. multi-site) stimulation techniques and, in particular, for different topologies of the connectivity (Hauptmann et al., 2005; Popovych et al., 2006; Popovych and Tass, 2010). Remarkably, desynchronizing effects do not only propagate between neuronal populations in case of a connectivity pattern with a Gaussian connection probability (Hauptmann et al., 2005), but even with a mean field coupling, i.e. a connectivity pattern where each neuron of one of the populations is coupled to all neurons of the other population with equal strength in a uni-directional or even bi-directional setup (Popovych et al., 2006; Popovych and Tass, 2010). These results indicate that the propagation of desynchronizing effects might be a robust mechanism which does not depend on the type of the stimulation technique causing the desynchronization in an upstream population. Mechanism (ii) might be particularly relevant for the propagation of desynchronizing effects from auditory to non-auditory areas, since it does not require a tonotopic organization of the connections.

In other words, the desynchronization at the level of the primary auditory cortex might be induced by the genuine CR mechanism, time-shifted phase resets of neuronal subpopulations (Tass, 2003), or by a propagation of desynchronizing effects from an upstream nucleus of the central auditory system. In the latter case the genuine CR mechanism takes place in the upstream nucleus. From a theoretical standpoint, combinations of mechanisms (i) and (ii) might also be conceivable. 
To study the dynamical mechanism of acoustic CR neuromodulation in tinnitus patients in more detail, in a forthcoming EEG study we shall analyze in which auditory and non-auditory brain areas single tones cause a phase reset of pathological rhythms (e.g. $\delta$ ) and CR stimuli (i.e. time-shifted sequences of phase resetting tones) cause a desynchronization.

\subsection{Pathophysiology of tinnitus}

One limitation of the pioneering MEG study by Weisz and coworkers (2005a), revealing pathologically enhanced $\delta$ and decreased $\alpha$ in tinnitus patients, was that contrary to the normal hearing control group the tinnitus group had a high-frequency hearing loss. Since the brain regions with increased $\delta$ power were also the regions of decreased $\alpha$ power, the results by Weisz et al. (2005a) are similar to findings obtained during slow-wave sleep (Benoit et al., 2000). Accordingly, Weisz et al. (2005a) could not rule out that the changes in spontaneous brain activity might simply be due to sensory deprivation, in terms of the highfrequency hearing loss, rather than being specific for tinnitus. In contrast, our EEG results were obtained in one group of patients with tinnitus and after significant CR induced tinnitus relief and having the same hearing levels before and after therapy. Accordingly, our study does not feature the limitation of a nonmatched hearing loss between comparison groups and, hence, substantially confirms the findings by Weisz et al. (2005a), in accordance, e.g., with the neurofeedback (Dohrmann et al., 2007a) and residual inhibition (Kahlbrock and Weisz, 2008) studies. Hyperacusis and depression were not assessed in our RESET study.

\subsection{Limitation of the RESET trial}

The RESET trial is an exploratory first in man trial and not a fully powered trial. It serves the purpose to collect safety information as well as efficacy data for different doses of acoustic CR. Therefore several shortcomings of the trial have to be taken into account: no confirmatory statistical approach was used but several efficacy endpoints common in tinnitus research were investigated with statistics of exploratory nature. EEG recordings were used as biomarkers to explore the effects of acoustic CR on different brain regions. Randomization was done using computer random codes. By chance and "small group effect" the allocation of patients to the placebo group (G5) showed significant differences in baseline scores concerning several important parameters. The small group size and the baseline difference of the placebo group is clearly a weak point of the trial. The main purpose of our placebo group was to have stimulation tones, that where sufficiently remote from the tinnitus frequency. This was motivated by few case studies of acoustic CR treatment performed prior to the start of the RESET trial. In these cases we observed that shifting all CR tones by around $50 \%$ to either side of the tinnitus frequency and applied for several hours per day may have adverse effects (e.g. transient headache, transient increase of tinnitus loudness and annoyance). For this reason, to avoid side effects but nevertheless perform acoustic stimulation resembling therapeutic stimulation, in the placebo group we delivered CR tones far away from the range of tinnitus frequency for one hour per day only. Dosage based un-blinding of the placebo group was prevented by an active stimulation group (G4) with similar dosage (one hour per day).

Another important aspect in the context of unblinding is the on-stimulation effect. Remarkably, in accordance with our theoretical predictions (Tass and Hauptmann, 2009) the stimulation in G2 has on-stimulation effects, but no off-stimulation effects ("pseudo placebo").

However, additional analyses provide further evidence for the effectiveness of the CR treatment. Concerning the different baseline characteristics of the placebo group, on the one hand we showed that treatment success did not correlate with tinnitus duration, tinnitus severity or age. On the other hand, subgroups of G1 matched to the placebo group with respect to size and tinnitus severity were compared to the placebo group. We found a substantially greater improvement in G1 subgroups as compared to G5. Furthermore, to perform a statistical comparison between two groups with comparable sizes, we pooled two big groups: The "effective stimulation group" G1 + G3 $(n=34$, 4-6h/day CR neuromodulation) was compared with the "ineffective stimulation group" comprising all data of G2 ("noisy" stimulation with a masking effect but without lasting off-effect), G4 (only $1 \mathrm{~h}$ of CR neuromodulation per day leading to small improvements only) and G5 (the placebo group) with $n=29$ patients. Although the "ineffective stimulation group" showed significant results compared to baseline concerning VAS loudness and annoyance in the ON condition as well as TQ, the "effective stimulation group" was significantly better than the "ineffective stimulation 
group" with respect to VAS loudness on/off after 12 and 16 weeks, VAS annoyance on/off after 12 weeks, and TQ after 12 weeks. These results are accompanied by a pronounced shift in tinnitus frequency in the pooled CR neuromodulation group $(\mathrm{G} 1+\mathrm{G} 3)$. Finally, we performed a comparison between all groups and the active control group G2, the masker-like noisy CR group, which showed a good on-effect without lasting off-effect. G1 and G3, both groups with 4-6h/day of CR neuromodulation turned out to reveal significantly better results than the "masker like" group G2. Still the exploratory data from the RESET trial serve now for the start of a fully powered prospective multicenter double blind placebo controlled randomized trial ("RESET 2") that will randomize 100 patients with chronic tonal tinnitus either to pseudo placebo or acoustic CR.

Patients profited differently from the CR neuromodulation. In future studies, we aim to elucidate the influence of clinical or/and EEG variables prior to therapy on the therapy success.

Overall, our study strongly supports the pivotal role of pathological synchrony in tinnitus generation (Llinás et al., 1999; Weisz et al., 2005a; Dohrmann et al., 2007a; Kahlbrock and Weisz, 2008; van der Loo et al., 2009; Moazami-Goudarzi et al., 2010), as suggested, e.g., in the context of thalamocortical dysrhythmia (Llinás et al., 1999). Along with the results of the experimental studies of our group this approach may therefore open novel therapeutic and neuroscientific avenues.

\section{Acknowledgments}

We are grateful to Drs. N. Pasch, H. Hermes, D. Jürgens, J. Alberty, G. Krückels, W. Schütz, and S. Schütz for contributions to patient selection. We gratefully acknowledge discussions with Dr. Oleksandr Popovych and Dr. Timea Tóth. The RESET study was funded by Research Center Jülich and ANM Adaptive Neuromodulation GmbH.

\section{Conflict of interest}

Dr. Peter Tass and Dr. Christian Hauptmann have a contractual relationship with ANM Adaptive Neuromodulation $\mathrm{GmbH}$, the sponsor of the clinical trial. Dr. Peter Tass and Dr. Hans-Joachim Freund are holding shares of ANM Adaptive Neuromodulation $\mathrm{GmbH}$.

\section{References}

Benabid, A.L., Pollak, P., Gervason, C., Hoffmann, D., Gao, D.M., Hommel, M., Perret, J.E. \& de Rougemount, J. (1991). Longterm suppression of tremor by chronic stimulation of ventral intermediate thalamic nucleus. The Lancet, 337, 403-406.

Benoit, O., Daurat, A. \& Prado, J. (2000). Slow (0.7-2 Hz) and fast $(2-4 \mathrm{~Hz})$ delta components are differently correlated to theta, alpha and beta frequency bands during NREM sleep. Clin $\mathrm{Neu}$ rophysiol, 111, 2103-2106.

Caffier, P., Haupt, H., Scherer, H. \& Mazurek, B. (2006). Outcomes of long-term outpatient tinnitus-coping therapy: psychometric changes and value of tinnitus-control instruments. Ear Hear, 27, 619-627.

Chen, R., Classen, J., Gerloff, C., Celnik, P., Wassermann, E.M., Hallett, M. \& Cohen, L.G. (1997). Depression of motor cortex excitability by low-frequency transcranial magnetic stimulation. Neurology, 48, 1398-1403.

De Ridder, D., Verstraeten, E., Van der Kelen, K., De Mulder, G., Sunaert, S., Verlooy, J., Van de Heyning, P. \& Moller, A.R. (2005). Transcranial magnetic stimulation for tinnitus: Influence of tinnitus duration on stimulation parameter choice and maximal tinnitus suppression. Otol Neurootol, 26, 616-619.

De Ridder, D., De Mulder, G., Verstraeten, E., Van der Kelen, K., Sunaert, S., Smits, M., Kovacs, S., Verlooy, J., Van de Heyning, P. \& Moller, A.R. (2006). Primary and secondary auditory cortex stimulation for intractable tinnitus. ORL J Otorhinolaryngol Relat Spec, 68, 48-55.

De Ridder, D., De Mulder, G., Menovsky, T., Sunaert, S. \& Kovacs, S. (2007). Electrical stimulation of auditory and somatosensory cortices for treatment of tinnitus and pain. Prog Brain Res, 166(36), 377-388.

De Ridder, D., Van der Loo, E., Vanneste, S., Gais, S., Plazier, M., Kovacs, S., Menovsky, T. \& Van de Heyning, P. (2011). Theta-gamma dysrhythmia and auditory phantom perception. J Neurosurg, 114(4), 912-921.

De Ridder, D. \& Vanneste, S. (2011). Auditory Cortex Stimulation for Tinnitus. In: Moller, A.R., Langguth, B., De Ridder, D. \& Kleinjung, T. (Eds.), Textbook of Tinnitus, Springer, New York, 717-726.

Dietrich, V., Nieschalk, M., Stoll, W., Rajan, R. \& Pantev, C. (2001). Cortical reorganization in patients with high frequency cochlear hearing loss. Hear Res, 158, 95-101.

Dohrmann, K., Elbert, T., Schlee, W. \& Weisz, N. (2007a). Tuning the tinnitus percept by modification of synchronous brain activity. Restor Neurol Neurosci, 25, 371-378.

Dohrmann, K., Weisz, N., Schlee, W., Hartmann, T. \& Elbert, T. (2007b). Neurofeedback for treating tinnitus. Prog Brain Res, $166,473-485$.

Eggermont, J.J. (1992). Neural interaction in cat primary auditory cortex. Dependence on recording depth, electrode separation, and age. $J$ Neurophysiol, 68, 1216-1228. 
Eggermont, J.J. (2003). Central tinnitus. Auris Nasus Larynx, 30, 7-12.

Eggermont, J.J. \& Robert, L.E. (2004). The neuroscience of tinnitus. Trends Neurosci, 27, 676-682.

Ehret, G. \& Romand, R. (1997). (Eds.), The central auditory system, Oxford University Press, Oxford.

Elbert, T., Rockstroh, B., Kowalik, Z.J. \& Hoke, M. (1997). Clinical applications of EEG analyses using tools derived from nonlinear system theory. In: Angelieri, F., Butler, S.R., Giaquinto, S. \& Majkowski, J. (Eds.), Analysis of the electrical activity of the brain, Wiley and Sons, London.

Engineer, N.D., Riley, J.R., Seale, J.D., Vrana, W.A., Shetake, J.A., Sudanagunta, S.P., Borland, M.S. \& Kilgard, M.P. (2011). Reversing pathological neural activity using targeted plasticity. Nature, 470, 101-104.

Feldmann, H. (1971). Homolateral and contralateral masking of tinnitus by noise-bands and pure tones. Audiol (Basel), 10, 138-144.

Frank, G., Kleinjung, T., Landgrebe, M., Vielsmeier, V., Steffenhagen, C., Burger, J., Frank, E., Vollberg, G., Hajak, G. \& Langguth, B. (2010). Left temporal low-frequency rTMS for the treatment of tinnitus: Clinical predictors of treatment outcome - a retrospective study. Eur J Neurol, 17, 951-956.

Franosch, J.M., Kempter, R., Fastl, H. \& Van Hemmen, J.L. (2003). Zwicker tone illusion and noise reduction in the auditory system. Phys Rev Lett, 90, 178103.

Gerstner, W., Kempter, R., Van Hemmen, L. \& Wagner, H. (1996). A neuronal learning rule for sub-millisecond temporal coding. Nature, 383, 76-81.

Goebel, G. \& Hiller, W. (1994). The tinnitus questionnaire. A standard instrument for grading the degree of tinnitus. Results of a multicenter study with the tinnitus questionnaire. $H N O, 42$, 166-172.

Goebel, G. \& Hiller, W. (1998). Tinnitus-Fragebogen (TF). Ein Instrument zur Erfassung von Belastung und Schweregrad bei Tinnitus. Handanweisung, Göttingen, Hogrefe.

Goebel, G., Kahl, M., Arnold, W. \& Fichter, M. (2006). 15-year prospective follow-up study of behavioral therapy in a large sample of in patients with chronic tinnitus. Acta Otolaryngol Suppl, 556, 70-79.

Guttman, R., Lewis, S. \& Rinzel, J. (1980). Control of repetitive firing in squid axon membrane as a model for a neuroneoscillator. J Physiol, 305, 377-395.

Hämäläinen, M., Hari, R., Ilmoniemi, R.J., Knuutila, J. \& Lounasmaa, O.V. (1993). Magnetoencephalography-theory, instrumentation, and applications to noninvasive studies of the working human brain. Rev Mod Phys, 65, 413-497.

Hartmann, T., Lorenz, I. \& Weisz, N. (2011). Neurobiofeedback. In: Moller, A.R., Langguth, B., De Ridder, D. \& Kleinjung, T. (Eds.), Textbook of Tinnitus, Springer, New York, 691-696.

Hauptmann, C., Popovych, O. \& Tass, P.A. (2005). Effectively desynchronizing deep brain stimulation based on a coordinated delayed feedback stimulation via several sites: A computational study. Biol Cybern, 93, 463-470.

Hauptmann, C. \& Tass, P.A. (2007). Therapeutic rewiring by means of desynchronizing brain stimulation. Biosystems, 89, 173-181.
Hauptmann, C. \& Tass, P.A. (2009). Cumulative and after-effects of short and weak coordinated reset stimulation: A modeling study. J Neural Eng, 6, 016004.

Hauptmann, C. \& Tass, P.A. (2010). Restoration of segregated, physiological neuronal connectivity by desynchronizing stimulation. J Neural Eng, 7, 056008.

Hazell, J.W.P. \& Wood, S. (1981). Tinnitus masking - a significant contribution to tinnitus management. Brit J Audiol, 15, 223-230.

Hesser, H., Weise, C., Rief, W. \& Andersson, G. (2011). The effect of waiting: A meta-analysis of wait-list control groups in trials for tinnitus distress. J Psychosom Res, 70(4), 378-384.

Irvine, D.R., Rajan, R. \& Brown, M. (2001). Injury- and use-related plasticity in adult auditory cortex. Audiol Neurootol, 6, 192-195.

Jacobson, N.S. \& Truax, P. (1991). Clinical significance: A statistical approach to defining meaningful change in psychotherapy research. J Consult Clin Psychol, 59, 12-19.

Jastreboff, P.J. \& Hazell, J.W. (1993). A neurophysiological approach to tinnitus: Clinical implications. Br J Audiol, 27(1), 7-17.

Jeanmonod, D., Magnin, M. \& Morel, A. (1996). Low-threshold calcium spike bursts in the human thalamus. Common physiopathology for sensory, motor and limbic positive symptoms. Brain, 119, 363-375.

Kahlbrock, N. \& Weisz, N. (2008). Transient reduction of tinnitus intensity is marked by concomitant reductions of delta band power. BMC Biol, 16(6), 4.

Klass, D.W. \& Daly, D.D. (1979). (Eds.), Current practice of clinical electroencephalography, Raven Press, New York.

Kleinjung, T., Langguth, B. \& Khedr, E. (2011). Transcranial magnetic stimulation. In: Moller, A.R., Langguth, B., De Ridder, D. \& Kleinjung, T. (Eds.), Textbook of Tinnitus, Springer, New York, 697-710.

Klimesch, W., Hanslmayr, S., Sauseng, P. \& Gruber, W.R. (2006). Distinguishing the evoked response from a phase reset: A comment to Mäkinen et al. Neuroimage, 26, 808-811.

Kovacs, S., Peeters, R., Smits, M., De Ridder, D., Van Hecke, P. \& Sunaert, S. (2006). Activation of cortical and subcortical auditory structures at $3 \mathrm{~T}$ by means of a functional magnetic resonance imaging paradigm suitable for clinical use. Invest Radiol, 41, 87-96.

Kühn, A.A., Kempf, F., Brücke, C., Doyle, L.G., Martinez-Torres, I., Pogosyan, A., Trottenberg, T., Kupsch, A., Schneider, G.H., Hariz, M.I., Vandenberghe, W., Nuttin, B. \& Brown, P. (2008). High-frequency stimulation of the subthalamic nucleus suppresses oscillatory beta activity in patients with Parkinson's disease in parallel with improvement in motor performance. $J$ Neurosci, 28, 6165-6173.

Langguth, B., Eichhammer, P., Wiegand, R., Marienhagen, J., Maenner, P., Jacob, P. \& Hajak, G. (2003). Neuronavigated rTMS in a patient with chronic tinnitus. Effects of 4 weeks treatment. Neuroreport, 14, 977-980.

Liberman, M.C. \& Dodds, L.W. (1984). Single-neuron labeling and chronic cochlear pathology. III Stereocilia damage and alterations of threshold tuning curves. Hear Res, 16, 55-74.

Llinás, R.R., Ribary, U., Jeanmonod, D., Kronberg, E. \& Mitra, P.P. (1999). Thalamocortical dysrhythmia: 
A neurological and neuropsychiatric syndrome characterized by magnetoencephalography. Proc Natl Acad Sci US A, 96, 15222-15227.

Lockwood, A.H., Salvi, R.J., Coad, M.L., Towsley, M.L., Wack, D.S. \& Murphy, B.W. (1998). The functional neuroanatomy of tinnitus: Evidence for limbic system links and neural plasticity. Neurology, 50, 114-120.

Lockwood, A.H., Salvi, R.J., Burkard, R.F., Galantowicz, P.J., Coad, M.L. \& Wack, D.S. (1999). Neuroanatomy of tinnitus. Scand Audiol Suppl, 51, 47-52.

Lockwood, A.H., Salvi, R.J. \& Burkard, R.F. (2002). Tinnitus. N Engl J Med, 347, 904-910.

Lysyansky, B., Popovych, O.V. \& Tass, P.A. (2011). Desynchronizing anti-resonance effect of the m:n ON-OFF coordinated reset stimulation. J Neural Eng, 8, 036019.

Markram, H., Lübke, J., Frotscher, M. \& Sakmann, B. (1997). Regulation of synaptic efficacy by coincidence of postsynaptic APs and EPSPs. Science, 275, 213-215.

Martinez-Devesa, P., Perera, R., Theodoulou, M. \& Waddell, A. (2010). Cognitive behavioural therapy for tinnitus. Cochrane Database Syst Rev, 8(9), 1-33.

Melcher, J.R., Sigalovsky, I.S., Guinan, J.J. Jr. \& Levine, R.A. (2000). Lateralized tinnitus studied with functional magnetic resonance imaging: Abnormal inferior colliculus activation. $J$ Neurophysiol, 83, 1058-1072.

Moazami-Goudarzi, M., Michels, L., Weisz, N. \& Jeanmonod, D. (2010). Temporo-insular enhancement of EEG low and high frequencies in patients with chronic tinnitus. QEEG study of chronic tinnitus patients. BMC Neurosci, 11, 40.

Moller, A.R. (2003). Pathophysiology of tinnitus. Otolaryngol Clin North Am, 36, 249-266.

Moller, A.R. (2007). The role of neural plasticity in tinnitus. Prog Brain Res, 166, 37-45.

Moller, A.R., Langguth, B., De Ridder, D. \& Kleinjung, T. (2011), (Eds.), Textbook of tinnitus, Springer, New York.

Mühlnickel, W., Elbert, T., Taub, E. \& Flor, H. (1998). Reorganization of auditory cortex in tinnitus. Proc Natl Acad Sci U S A $95,10340-10343$

Neiman, A.B., Russell, D.F., Yakusheva, T.A., DiLullo, A. \& Tass, P.A. (2007). Response clustering in transient stochastic synchronization and desynchronization of coupled neuronal bursters. Phys Rev E Stat Nonlin Soft Matter Phys, E76, 021908.

Nichols, T.E. \& Holmes, A.P. (2002). Nonparametric permutation tests for functional neuroimaging: A primer with examples. Hum Brain Mapp, 15, 1-25.

Niedermeyer, E. \& Lopes da Silva, F.H. (1999), (Eds.), Electroencephalography. Basic principals, clinical applications, and related fields. 4th ed., Williams \& Wilkins, London.

Norena, A., Micheyl, C., Chery-Croze, S. \& Collet, L. (2002). Psychoacoustic characterization of the tinnitus spectrum: Implications for the underlying mechanisms of tinnitus. Audiol Neurootol, 7, 358-369.

Norena, A.J. \& Eggermont, J.J. (2003). Changes in spontaneous neural activity immediately after an acoustic trauma: Implications for neural correlates of tinnitus. Hear Res, 183, 137-153.
Nunez, P.L. (1981). Electric fields of the brain: The neurophysics of EEG, Oxford University Press, New York.

Ochi, K. \& Eggermont, J.J. (1997). Effects of quinine on neural activity in cat primary auditory cortex. Hear Res, 105, 105-118.

Okamoto, H., Stracke, H., Stoll, W. \& Pantev, C. (2010). Listening to tailor-made music reverses maladaptive auditory cortex reorganization and alleviates tinnitus. Proc Natl Acad Sci U S A, 107(3), 1207-1210.

Ortmann, M., Müller, N., Schlee, W. \& Weisz, N. (2011). Rapid increases of gamma power in the auditory cortex following noise trauma in humans. Eur J Neurosci, 33, 568-575.

Pascual-Leone, A., Valls-Solé, J., Wassermann, E.M. \& Hallett, M. (1994). Responses to rapid-rate transcranial magnetic stimulation of the human cortex. Brain, 117(4), 847-858.

Pascual-Marqui, R.D. (2002). Standardized low resolution brain electromagnetic tomography (sLORETA): Technical details. Methods Find Exp Clin Pharmacol, 24, 5-12.

Pickles, J.O. (1984). Frequency threshold curves and simultaneous masking functions in high-threshold, broadly-tuned, fibres of the guinea pig auditory nerve. Hear Res, 16, 91-99.

Plewnia, C., Bartels, M. \& Gerloff, C. (2003). Transient suppression of tinnitus by transcranial magnetic stimulation. Ann Neurol, 53, 263-266.

Popovych, O.V., Hauptmann, C. \& Tass, P.A. (2006). Desynchronization and decoupling of interacting oscillators by nonlinear delayed feedback. Int J Bif Chaos, 16, 1977-1987.

Popovych, O.V. \& Tass, P.A. (2010). Synchronization control of interacting oscillatory ensembles by mixed nonlinear delayed feedback. Phys Rev E, 82, 026204.

Popovych, O.P. \& Tass, P.A. (2011). Macroscopic entrainment of periodically forced oscillatory ensembles. Prog Biophys Mol Biol, 105, 98-108.

Popovych, O.V. \& Tass, P.A. (2012). Desynchronizing electrical and sensory coordinated reset neuromodulation. Front Hum Neurosci, 6, 58. doi:10.3389/fnhum.2012.00058

Quiroga, R.Q., Kraskov, A., Kreuz, T. \& Grassberger, P. (2002). Performance of different synchronization measures in real data: A case study on electroencephalographic signals. Phys Rev E, 65, 041903.

Rajan, R. \& Irvine, D.R. (1998). Neuronal responses across cortical field A1 in plasticity induced by peripheral auditory organ damage. Audiol Neurootol, 3, 123-144.

Rauschecker, J.P. (1999). Auditory cortical plasticity: A comparison with other sensory systems. Trends Neurosci, 22, 74-80.

Rauschecker, J.P., Leaver, A.M. \& Mühlau, M. (2010). Tuning out the noise: Limbic-auditory interactions in tinnitus. Neuron, 66 , 819-826.

Roberts, L.E., Eggermont, J.J., Caspary, D.M., Shore, S.E., Melcher, J.R. \& Kaltenbach, J.A. (2010). Ringing Ears: The Neuroscience of Tinnitus. J Neurosci, 30(45), 1497214979.

Robertson, D. \& Irvine, D.R. (1989). Plasticity of frequency organization in auditory cortex of guinea pigs with partial unilateral deafness. J Comp Neurol, 282, 456-471. 
Ryan, A., Dallos, P. \& McGee, T. (1979). Psychophysical tuning curves and auditory thresholds after hair cell damage in the chinchilla. J Acoust Soc Am, 66, 1096-1098.

Saunders, J.C. (2007). The role of central nervous system plasticity in tinnitus. J Commun Disord, 40, 313-334.

Scherg, M., Ille, N., Bornfleth, H. \& Berg, P. (2002). Advanced tools for digital EEG review: Virtual source montages, whole-head mapping, correlation, and phase analysis. J Clin Neurophysiol, 19(2), 91-112.

Schlee, W., Mueller, N., Hartmann, T., Keil, J., Lorenz, I. \& Weisz, N. (2009a). Mapping cortical hubs in tinnitus. BMC Biol, 7, 80.

Schlee, W., Hartmann, T., Langguth, B. \& Weisz, N. (2009b). Abnormal resting-state cortical coupling in chronic tinnitus. $B M C$ Neuroscience, 10, 11.

Seki, S. \& Eggermont, J.J. (2003). Changes in spontaneous firing rate and neural synchrony in cat primary auditory cortex after localized tone-induced hearing loss. Hear Res, 180(1-2), 28-38.

Smits, M., Kovacs, S., De Ridder, D., Peeters, R.R., Van Hecke, P. \& Sunaert, S. (2007). Lateralization of functional magnetic resonance imaging (fMRI) activation in the auditory pathway of patients with lateralized tinnitus. Neuroradiology, 49, 669-679.

Steriade, M. (2006). Grouping of brain rhythms in corticothalamic systems. Neuroscience, 137, 1087-1106.

Suckfüll, M., Althaus, M., Ellers-Lenz, B., Gebauer, A., Görtelmeyer, R., Jastreboff, P.J., Moebius, H.J., Rosenberg, T., Russ, H., Wirth, Y. \& Krueger, H. (2011). A randomized, double-blind, placebo-controlled clinical trial to evaluate the efficacy and safety of neramexane in patients with moderate to severe subjective tinnitus. BMC Ear Nose Throat Disord, 11(11), 1 .

Surr, R.K., Montgomery, A.A. \& Mueller, H.G. (1985). Effect of amplification on Tinnitus among new hearing aid users. Ear Hear, 6(2), 71-75.

Tass, P.A. (2002). Desynchronization of brain rhythms with soft phase-resetting techniques. Biol Cybern, 87, 102-115.

Tass, P.A. (2003). A model of desynchronizing deep brain stimulation with a demand-controlled coordinated reset of neural subpopulations. Biol Cybern, 89, 81-88.

Tass, P.A. \& Majtanik, M. (2006). Long-term anti-kindling effects of desynchronizing brain stimulation: A theoretical study. Biol Cybern, 94, 58-66.

Tass, P.A. \& Hauptmann, C. (2009). Anti-kindling achieved by stimulation targeting slow synaptic dynamics. Rest Neurol Neurosci, 27, 589-609.

Tass, P.A., Silchenko, A., Hauptmann, C., Barnikol, U.B. \& Speckmann, E.-J. (2009). Long-lasting desynchronization in rat hippocampal slice induced by coordinated reset stimulation. Phys Rev E Stat Nonlin Soft Matter Phys, 80, 011902.

Tass, P.A. \& Popovych, O.P. (2012). Unlearning tinnitus-related cerebral synchrony with acoustic coordinated reset stimulation - theoretical concept and modelling. Biol Cybern, 106, 27-36. doi: 10.1007/s00422-012-0479-5

Temperli, P., Ghika, J., Villemure, J.-G., Burkhard, P.R., Bogousslavsky, J. \& Vingerhoets, F.J.G. (2003). How do parkinsonian signs return after discontinuation of subthalamic DBS? Neurology, $60,78-81$.

Terry, A.M., Jones, D.M., Davis, B.R. \& Slater, R. (1983). Parametric studies of tinnitus masking and residual inhibition. Br J Audiol, $17,245-256$.

Tyler, R.S. (2006). Tinnitus: Standard of care, personality differences, genetic factors. ORL J Otorhinolaryngol Relat Spec, 68(1), 14-19.

Tyler, R.S. (2007). Clinical trials for tinnitus: Study populations, designs, measurement variables, and data analysis Prog Brain Res, 166, 499-509.

Van der Loo, E., Gais, S., Congedo, M., Vanneste, S., Plazier, M., Menovsky, T., Van de Heyning, P. \& De Ridder, D. (2009). Tinnitus intensity dependent gamma oscillations in the contralateral auditory cortex. PLoS One, 4, e7396.

Vanneste, S., Plazier, M., Van der Loo, E., De Heyning, P., Congedo, M. \& De Ridder, D. (2010). The neural correlates of tinnitusrelated distress. Neuroimage, 52(2), 470-480.

Vanneste, S., Van de Heyning, P. \& De Ridder, D. (2011). The neural network of phantom sound changes over time: A comparison between recent-onset and chronic tinnitus patients. Europ $J$ Neuroscience, 34(5), 718-731.

Wang, H., Wang, X. \& Scheich, H. (1996). LTD and LTP induced by transcranial magnetic stimulation in auditory cortex. $\mathrm{Neu}$ roreport, 7, 521-525.

Weisz, N., Moratti, S., Meinzer, M., Dohrmann, K. \& Elbert, T. (2005a). Tinnitus perception and distress is related to abnormal spontaneous brain activity as measured by magnetoencephalography. PLoS Med, 2(6), 546-553.

Weisz, N., Wienbruch, C., Dohrmann, K. \& Elbert, T. (2005b). Neuromagnetic indicators of auditory cortical reorganization of tinnitus. Brain, 128, 2722-2731.

Weisz, N., Hartmann, T., Dohrmann, K., Schlee, W. \& Norena, A. (2006). High-frequency tinnitus without hearing loss does not mean absence of deafferentation. Hear Res, 222, 108-114.

Weisz, N., Müller, S., Schlee, W., Dohrmann, K., Hartmann, T. \& Elbert, T. (2007). The neural code of auditory phantom perception. J Neurosci, 27, 1479-1484.

Wightman, F.L., McGee, T. \& Kramer, M. (1977) Factors influencing frequency selectivity in normal and hearing-impaired listeners. In: Evans, E.F. \& Wilson, J.P. (Eds.), Psychophysics and Physiology of Hearing, Academic Press, London.

Winfree, A.T. (1977). Phase control of neural pacemakers. Science, 197, 761-763.

Yang, S., Weiner, B.D., Zhang, L.S., Cho, S.J. \& Bao, S. (2011). Homeostatic plasticity drives tinnitus perception in an animal model. PNAS 108, 14974-14979.

Yuste, R. \& Bonhoeffer, T. (2004). Genesis of dendritic spines: Insights from ultrastructural and imaging studies. $\mathrm{Nat} \mathrm{Rev} \mathrm{Neu-}$ rosci, 5, 24-34.

Zwicker, E. (1964). "Negative Afterimage" in hearing. J Acoust Soc Am, 36, 2413. 